\title{
Sprachgebrauch und Spracheinstellungen in der Deutschschweiz. Pfarrpersonen als sprachbewusste Sprecherinnen und Sprecher im Fokus*
}

Susanne Oberholzer (Salzburg)

\begin{abstract}
The relation between Swiss German dialects and Standard German has been subject to public and scholarly debates for over 100 years. Among the most frequently discussed points are the appropriateness of spoken Standard German in different contexts and the attitudes of Swiss people towards the two varieties. This paper summarises the results of a completed research project (Oberholzer in Vorb.), which surveyed language use and language attitudes in Switzerland for a specific group of speakers: pastors and priests working in German-speaking Switzerland.

The paper shows how pastors and priests make use of the diglossic situation and the possibility to code-switch in Sunday services. The use of Standard German emerges as an important communicative resource in German-speaking Switzerland. In addition, real language use and intended language use match to a high degree; this shows the degree of language awareness of this particular group in a diglossic situation.

Furthermore, a relatively new approach - the assumption that several mental models of High German coexist - helps to show differentiated language attitudes and to contradict some of the most common stereotypes regarding Standard German in German-speaking Switzerland. The attitudes towards Standard German in this study are significantly more positive than those observed in previous studies.
\end{abstract}

\section{$1 \quad$ Einleitung}

In der Deutschschweiz ist das Verhältnis zwischen den schweizerdeutschen Dialekten („Schweizerdeutsch“) und Standarddeutsch seit über hundert Jahren ein Thema, das sowohl die Wissenschaft als auch die Öffentlichkeit interessiert und umtreibt. Der Fokus der öffentlichen Diskussion liegt dabei unter anderem auf der Frage, welche Rolle Standarddeutsch in der Deutschschweiz spielen soll bzw. für welche Kontexte Standarddeutsch als gesprochene Sprache angemessen ist. In den letzten Jahren geriet dabei beispielsweise der Kontext Kindergarten und Volksschule in den Brennpunkt und die Frage, ob bereits im Kindergarten

\footnotetext{
* Für seine kritischen und wertvollen Hinweise zu diesem Beitrag danke ich Manfred Glauninger. Darüber hinaus bedanke ich mich bei Philipp Stoeckle für das Erstellen der Karten.

Linguistik online 85, 6/17 - http://dx.doi.org/10.13092/lo.85.4084
}

CC by 3.0 
Standarddeutsch gesprochen und in der Primarschule konsequent Standarddeutsch als Unterrichtssprache verwendet werden solle. ${ }^{1}$

Diese Diskussion liegt in der Sprachsituation der Deutschschweiz begründet, in der die schweizerdeutschen Dialekte in erster Linie der mündlichen Kommunikation dienen, während Standarddeutsch vor allem für schriftliche Kontexte verwendet wird. Traditionell wird diese sprachliche Realität als „Diglossie“ beschrieben (cf. z. B. Haas 2004, Sieber 2010, Petkova 2012). Jedoch werden gerade auch in jüngerer Vergangenheit Stimmen laut, die die Sprachsituation eher als eine ,bilinguale“ bezeichnen. Dies geschieht unter anderem aufgrund der Annahme, dass Standarddeutsch für Deutschschweizerinnen und Deutschschweizer in deren Selbsteinschätzung eine Fremdsprache darstelle (cf. Ris 1990: 43, Berthele 2004: 127).

Den Fragen, welche Einstellungen eine ausgewählte Gruppe von Deutschschweizerinnen und Deutschschweizern zu Standarddeutsch (und Schweizerdeutsch) hat, ob sie Standarddeutsch tatsächlich als Fremdsprache einschätzt und wie sie die beiden Varietäten in ihrem Alltag verwendet, war eine Studie (Oberholzer in Vorb.) gewidmet, deren wichtigste Resultate im vorliegenden Beitrag vorgestellt werden. Es handelt sich um Pfarrpersonen der beiden grossen Landeskirchen $^{2}$ der Deutschschweiz (der Evangelisch-reformierten und der Römischkatholischen), die in ihrem Berufsalltag sowohl den Dialekt als auch Standarddeutsch mündlich verwenden. Die Pfarrerinnen und Pfarrer sind eine Art Berufssprecherinnen und -sprecher und können aufgrund ihrer Ausbildung sowie der beruflichen Tätigkeit als besonders sprachaffine und sprachbewusste Sprecherinnen und Sprecher gelten.

Kirchen bilden einen der „Gebrauchskontexte“, wo der Standardgebrauch in der Deutschschweiz „situationsinduziert“3 ist: Es handelt sich um Kontexte, „die sich durch hohe Formalität und Distanz auszeichnen oder für welche die Standardsprache institutionalisiert ist" (Christen et al. 2010: 13). Es ist diese Art von Standardsprechen, die

aus der Perspektive der Sprechergemeinschaft prominent ins Bewusstsein kommt, wenn es um die Präferenz der gesprochenen Standardsprache im Deutschschweizer Sprachleben geht. Es handelt sich um einen Gebrauch der Standardsprache, mit dem die gesamte Deutschschweizer Bevölkerung selbstverständlich rezeptiven Umgang hat, in deren Produktion aber nur ein geringer Teil involviert ist.

(Christen et al. 2010: 13f.)

Pfarrerinnen und Pfarrer sind in diese Art von Standarddeutsch-Produktion involviert. Da in ihrem Berufsalltag, auch im Gottesdienst, der Dialekt aber ebenfalls eine mögliche Varietät für die Mündlichkeit ist, stellt sich die Frage, wie die Pfarrpersonen mit der Varietätenvielfalt umgehen und wie sie die beiden Varietäten einschätzen. Antworten auf diese Fragen gibt die vorgestellte Studie; die wichtigsten Ergebnisse werden im Folgenden vorgestellt.

\footnotetext{
1 Cf. dazu z. B. aus wissenschaftlicher Sicht Bachmann/Good (2003), Bachmann/Sigg (2004), Berthele (2010), Gyger (2005, 2007), Oberholzer (2006). Die Diskussion findet aber auch in den Medien statt, cf. z. B. Schneebeli (2008), Minor (2010), Häcki Buhofer (2011), Kalberer (2010), Brönnimann (2011), Wüthrich (2011).

2 Für die Untersuchung nicht berücksichtigt wurde die dritte Landeskirche der Schweiz, die christkatholische Kirche. Diese ist mit 33 Kirchgemeinden und rund 13'100 Mitgliedern (Stand Eidgenössische Volkszählung 2000, cf. von Arx 2010) die kleinste der drei Schweizer Landeskirchen.

3 Christen et al. (2010: 13f.) unterscheiden drei mögliche Konstellationen: „situationsinduziert“, ,,adressateninduziert" und ,diskursinduziert".
} 
In Kapitel 2 wird zunächst der Forschungsgegenstand definiert. Anschliessend werden die Forschungsfragen und die drei wichtigsten Methoden vorgestellt, mit denen die Daten für die Untersuchung erhoben wurden (Kapitel 3). Kapitel 4 ist den Resultaten gewidmet: Es wird zunächst gezeigt, wie Dialekt und Standarddeutsch in Gottesdiensten der beiden Kirchen eingesetzt werden, um anschliessend einen Blick darauf zu werfen, wie die Pfarrpersonen selbst ihren Sprachgebrauch einschätzen. Abschliessend wird gezeigt, dass es zu kurz greift, die Spracheinstellungen von Deutschschweizerinnen und Deutschschweizern zu Standarddeutsch auf das Fremdsprachen-Stereotyp zu reduzieren: Einstellungen zu Dialekt und Standarddeutsch sind mehrdimensional und zeigen - zumindest für die hier untersuchte Sprechergruppe - ein weitaus positiveres Bild von Standarddeutsch, als dies einige bisherige Untersuchungen (z. B. Scharloth 2005) tun. In Kapitel 5 werden die wichtigsten Punkte zusammengefasst und es wird ein Ausblick präsentiert.

\section{Die Deutschschweizer Kirchen, deren Pfarrpersonen und die Varietätenwahl}

Zur Varietätenwahl in den Kirchen der Deutschschweiz gab es in der Vergangenheit kaum empirische Untersuchungen. Ausnahmen bilden die Studien von Rüegger et al. (1996) und Schwarzenbach (1969). Erstere arbeitete mit objektiven Daten; für diese wurden Tonaufnahmen von reformierten Gottesdiensten im Kanton Zürich untersucht (mit klarem Fokus auf der Predigt). Schwarzenbach hingegen analysierte mittels einer Fragebogenerhebung subjektive Daten. Darüber hinaus gibt es diverse subjektive Schilderungen bzw. Einschätzungen der sprachlichen Situation in den Kirchen der Deutschschweiz sowohl von Seiten von Linguistinnen und Linguisten (z. B. Haas 2000, Lötscher 1983, Rash 1998, Siebenhaar/Wyler 1997, Sieber/Sitta 1986) als auch von Seite der Theologinnen und Theologen (z. B. Bietenhard 1990, Koch 1990, Meili 1985, Rickenmann 2005, Urwyler/Gysel 2011). Gemeinhin wird in diesen Texten davon ausgegangen, dass die Verwendung von Dialekt in den Gottesdiensten zugenommen hat. So schreibt beispielsweise Rash (1998: 67): "in practice, sermons in both Catholic and Protestant churches are increasingly held in dialect”. Schläpfer (1994: 298) hielt vier Jahre davor fest, dass „die Mundart in der Kirche die Standardsprache verdrängt“ habe, und zwar ,[f]ast noch deutlicher als in der Schule“.

Die vorliegende Studie widmete sich dem Sprachgebrauch und den Spracheinstellungen von Pfarrpersonen der beiden grossen Landeskirchen: also Pfarrerinnen und Pfarrer der Evangelisch-reformierten Kirche (reformierte Kirche) und Priestern der Römischkatholischen Kirche (katholische Kirche). Zur reformierten Kirche bekennen sich gemäss Strukturerhebung 2014 (Bundesamt für Statistik 2016) 25.5\% der Schweizer Wohnbevölkerung, zur römisch-katholischen $37.9 \%{ }^{4}$ Im Zentrum der Untersuchung stehen die Pfarrpersonen der reformierten Kirche. Diese Fokussierung ist in der Unterschiedlichkeit der Gottesdienste der beiden Kirchen begründet: Reformierte Pfarrpersonen können einen grossen Teil der Texte, die sie im Gottesdienst sprechen, selbst formulieren; katholische Priester hingegen sind beim freien Formulieren eigener Texte weit eingeschränkter: Durch Ordinarium und Proprium sind viele Texte vorgegeben. Aufgrund der Annahme, dass der sprachliche Gestaltungsspielraum grösser ist, wenn weniger standarddeutsche Textvorlagen

\footnotetext{
${ }^{4}$ Bei den Schweizer Bürgerinnen und Bürgern sind es $31.8 \%$, d. h. rund 1.66 Millionen Personen, die sich zur reformierten Kirche bekennen, und 37.3 \% bzw. 1.95 Millionen, die sich als katholisch bezeichnen.
} 
bestehen, die abgelesen (oder auswendig nachgesprochen) werden können, bietet sich der reformierte Gottesdienst als Untersuchungsgegenstand für eine Studie zum Gebrauch von Dialekt und Standarddeutsch stärker an.

Als Untersuchungsgebiet dienten für die reformierte Kirche fünf Kantone der Deutschschweiz: Basel-Landschaft, Bern, Thurgau, St. Gallen und Zürich (cf. Abb. 1). In diesen wurden Aufnahmen von 20 reformierten Gottesdiensten gemacht und anschliessend die Pfarrpersonen in einem Tiefeninterview befragt. Zusätzlich wurde bei allen reformierten Pfarrerinnen und Pfarrern eine Onlinefragebogenerhebung durchgeführt, um gewisse Resultate aus den Interviews durch ein breiter abgestütztes Sample zu validieren.

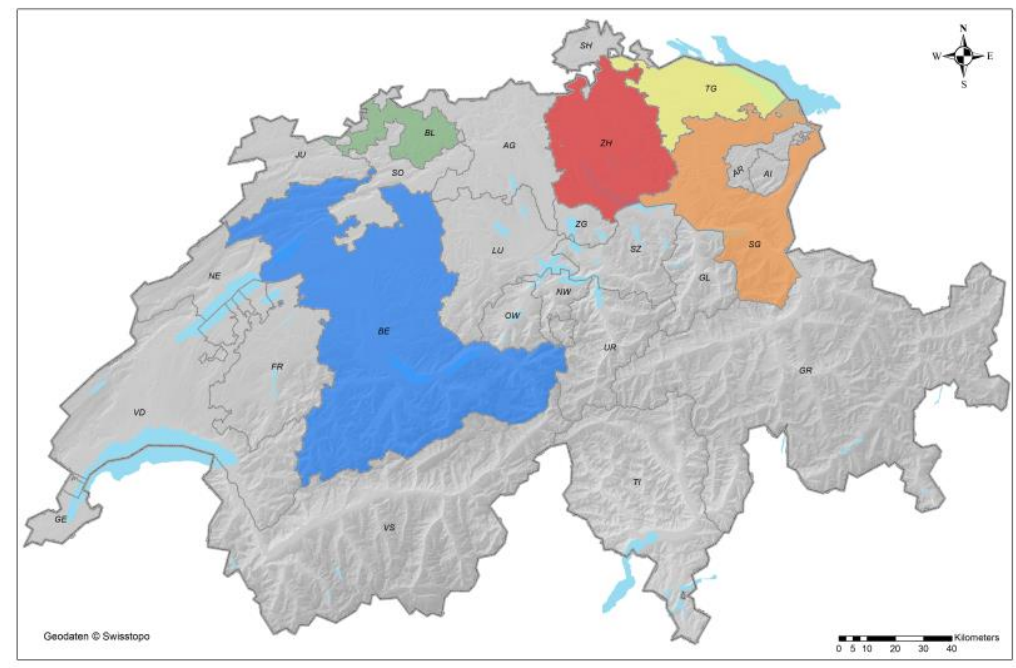

Abbildung 1: Erhebungsgebiet für die reformierte Kirche: Thurgau (gelb), Zürich (rot), St. Gallen (orange), Basel-Landschaft (grün) und Bern (blau). Karte: Philipp Stoeckle

Die sechs Aufnahmen von katholischen Gottesdiensten und die anschliessenden Tiefeninterviews wurden in den drei Bistümern der Deutschschweiz gemacht: Basel, Chur und St. Gallen (cf. Abb. 2).

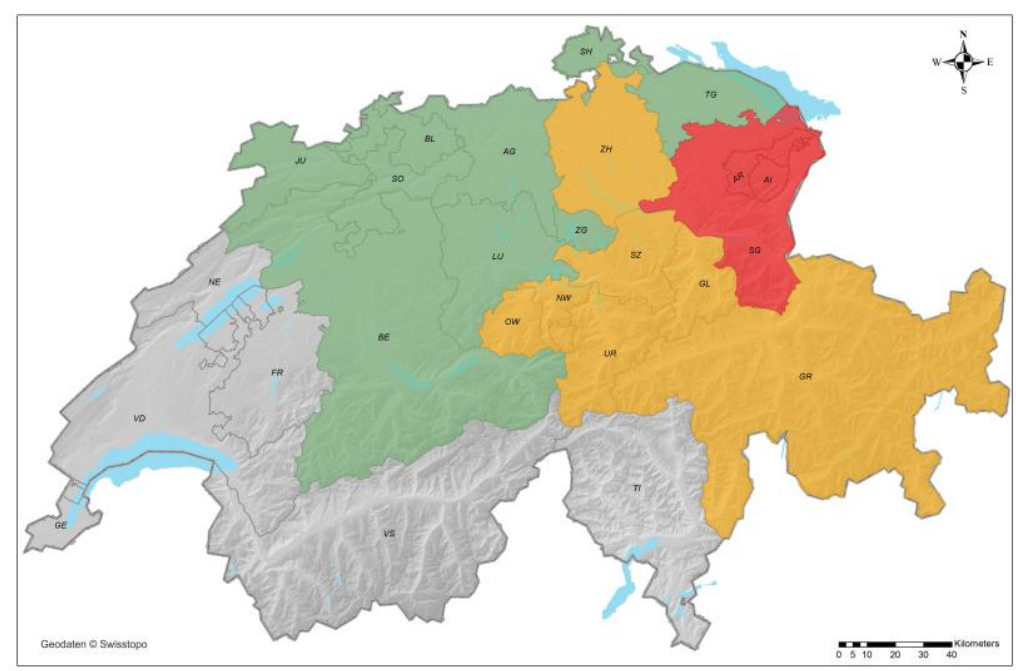

Abbildung 2: Erhebungsgebiet für die katholische Kirche: Bistum Basel (grün), Bistum Chur (gelb), Bistum St. Gallen (rot). Karte: Philipp Stoeckle 


\section{Forschungsfragen und Methoden}

Die zentralen Forschungsfragen, die das Projekt leiteten, waren die folgenden:

1. Intendierter Varietätengebrauch im Berufsalltag: Welche Varietäten verwenden Pfarrerinnen und Pfarrer in ihrem Berufsalltag gemäss eigener Aussage: in Gottesdiensten, im Religionsunterricht, für Kasualien, ${ }^{5}$ für Seelsorgegespräche? Wie begründen sie die Wahl der jeweiligen Varietät?

2. Tatsächlicher Varietätengebrauch im Sonntagsgottesdienst: Welche Faktoren beeinflussen die Varietätenwahl? An welchen Stellen im Gottesdienst finden allfällige Varietätenwechsel statt? Lassen sich diese kategorisieren?

3. Einstellungen zu Dialekt und Standarddeutsch: Welche Spracheinstellungen haben Pfarrerinnen und Pfarrer dem Dialekt und dem Standarddeutschen („Hochdeutschen“) gegenüber? Besteht zwischen den geäusserten Einstellungen und dem Sprachverhalten ein Zusammenhang?

Weitere Fragen, denen im Projekt nachgegangen wurde, waren jene nach möglichen Vorschriften bezüglich Varietäten vonseiten der reformierten Kantonalkirchen bzw. der katholischen Bistümer, jene nach der Varietätenfrage in der Aus- und Weiterbildung von Pfarrpersonen und jene nach der Rolle von Dialektvorlagen für die Gestaltung von Gottesdiensten. Im Folgenden wird der Fokus auf die Antworten zu den drei zentralen Fragestellungen gelegt und aufgezeigt, welche Varietäten die Pfarrpersonen im Gottesdienst verwenden, ob die tatsächliche Verwendung mit der subjektiven Einschätzung übereinstimmt und wie die beiden Varietäten in verschiedenen Kontexten beurteilt werden.

\subsection{Methodenmix zur Gewinnung objektiver und subjektiver Sprachdaten}

Um diese Fragen zu beantworten, wurden mittels eines Methodenmixes verschiedene subjektive und objektive Sprachdaten von reformierten und katholischen Pfarrpersonen erhoben. Folgende Methoden kamen dabei zum Einsatz:

- Gottesdienstaufnahmen

- Leitfadengesteuerte Interviews

- Onlinefragebogenerhebung

- Schriftliche Umfrage bei reformierter und katholischer Kirche

- Interview mit Professor für Praktische Theologie

Die ersten drei Methoden werden im Folgenden kurz einzeln präsentiert.

\footnotetext{
5 „Als Kasualien oder als Amtshandlungen werden die liturgisch geordneten kirchlichen Handlungen mit Ausnahme des sonntäglichen Gottesdienstes bezeichnet. Im Unterschied zum Gottesdienst werden die kasuellen Feiern nicht an regelmäßig wiederkehrenden Daten des Kalender- oder Kirchenjahres, sondern aus einem bestimmten Anlaß abgehalten. Sie beziehen sich auf eine einmalige und einzigartige Situation (Kasus) im Leben des einzelnen Christen oder im Lebenszusammenhang der christlichen Gemeinschaft.“" (Steck 1988: 673) Es handelt sich also beispielsweise um Hochzeiten, Beerdigungen und Taufen.
} 
Um authentische Sprachgebrauchsdaten zu erhalten und mögliche Varietätenwechsel in Gottesdiensten festzustellen, wurden Tonaufnahmen von 40 reformierten und 6 katholischen Sonntagsgottesdiensten untersucht. Die Aufnahmen der katholischen Gottesdienste sowie die Hälfte der reformierten Gottesdienste wurden von der Verfasserin für diese Studie erstellt. ${ }^{6}$ Dabei wurden in jedem der fünf ausgewählten Kantone für die reformierte Kirche je vier Sonntagsgottesdienste aufgezeichnet. In den drei Bistümern wurden jeweils zwei katholische Messen (mit Eucharistie) aufgenommen. Die übrigen 20 Aufnahmen (Vergleichsaufnahmen) von reformierten Gottesdiensten stammen von zwei Kirchgemeinden in den Kantonen Thurgau und St. Gallen und wurden der Verfasserin für die Studie zur Verfügung gestellt. Aus dem Pool dieser Aufnahmen wurden von vier Pfarrpersonen jeweils fünf Aufnahmen ausgewählt: So kann das Sprachverhalten dieser vier Pfarrpersonen über einen Einzelgottesdienst hinaus untersucht werden.

Für die Erhebung des intendierten Sprachgebrauchs sowie der Spracheinstellungen wurde mit allen Pfarrpersonen, von denen Gottesdienste aufgezeichnet und/oder analysiert worden sind, ein leitfadengesteuertes Tiefeninterview geführt. Dieses diente zusätzlich dazu, weitere Fragen zu beantworten, beispielsweise jene nach der Bedeutung der Sprachformenfrage während der Ausbildung zur Pfarrperson sowie jene nach der Relevanz von Dialektvorlagen (Dialektlieder, Dialektbibeln, Dialektliturgien).

Während die Tiefeninterviews mit 30 Pfarrpersonen (24 reformierte und 6 katholische) einen vertieften Einblick in das intendierte Sprachverhalten dieser spezifischen Gruppe ermöglichen, lassen sich daraus keine allgemeingültigen Aussagen ableiten. Daher wurden die Daten ergänzt durch die Antworten aus einer breit angelegten Onlinefragebogenerhebung, die ausschliesslich unter reformierten Pfarrerinnen und Pfarrern durchgeführt wurde. Diese wurde über www.soscisurvey.de durchgeführt. Bevor die Pfarrpersonen den Link per E-Mail bekamen, wurden sie von ihren Kantonalkirchen über die bevorstehende Onlinebefragung informiert und gebeten, daran teilzunehmen. Dies ist sicherlich eine Erklärung für die hohe Rücklaufquote von $60.2 \%$ im Durchschnitt (681 Antworten). ${ }^{7}$ Mittels Filterfragen wurden Gewährspersonen, deren Erstsprache ein schweizerdeutscher Dialekt ist, eruiert: 454 Pfarrpersonen wurden so als autochthone Sprecherinnen und Sprecher eines solchen ermittelt. Diese Antworten werden im Folgenden präsentiert. Der Anteil der Männer innerhalb des Samples liegt bei $64.5 \%$. Die Gewährspersonen waren zum Erhebungszeitpunkt im Durchschnitt 50 Jahre alt und arbeiteten durchschnittlich seit 18 Jahren im Pfarramt. Die Fragen fokussierten den intendierten Sprachgebrauch der Pfarrpersonen im Berufsalltag, die Einschätzung der persönlichen Entwicklung bei der Verwendung der Varietäten, den Gebrauch von Dialektvorlagen, die Rolle der Varietäten in der Ausbildung sowie die Verschriftung der Gottesdienstvorbereitung.

Das Korpus der vorliegenden Studie besteht also einerseits aus objektiven Sprachdaten in Form von Gottesdienstaufnahmen, die auf Code-Switchings bzw. auf die Verteilung der

\footnotetext{
${ }^{6}$ Es wurde ein Aufnahmegerät Zoom H2 mit einem daran angeschlossenen Richtmikrophon der Marke Sennheiser verwendet. Die Aufnahmen wurden im mp3-Format abgespeichert.

7 Zwischen $53.8 \%$ (Kanton Bern) und $72.0 \%$ (Thurgau). Darüber hinaus scheint ein genuines Interesse am Thema zu bestehen, erklärten doch von den 681 Personen, die den Fragebogen vollständig ausfüllten, $77.5 \%$, sie seien an den Resultaten der Studie interessiert und gaben zu diesem Zweck ihre E-Mail-Adresse an.
}

ISSN 1615-3014 
Varietäten untersucht wurden, andererseits aus subjektiven Daten aus leitfadengesteuerten Interviews mit 30 Pfarrpersonen und aus Antworten einer breit angelegten Onlinefragebogenerhebung, von denen 454 hier präsentiert werden.

\section{Varietätengebrauch und Einstellungen bei Deutschschweizer Pfarrpersonen}

Im Folgenden werden die wichtigsten Resultate der Studie vorgestellt. Dabei soll zunächst ein Blick auf die objektiven Sprachdaten, also die Daten von den Tonaufnahmen der Sonntagsgottesdienste, geworfen und die Frage beantwortet werden, an welchen Stellen des Gottesdienstes Varietätenwechsel stattfinden und ob sich diese kategorisieren lassen. Dies geschieht für die beiden Konfessionen getrennt, ein Vergleich von katholischen und reformierten Daten wird dabei aber nicht ausgespart. Anschliessend wird der Fokus auf die subjektiven Daten aus Tiefeninterviews und Onlinefragebögen gelegt und der Frage nachgegangen, welche Varietät die Pfarrpersonen gemäss eigener Aussage für ihre Gottesdienste verwenden. Der Fokus liegt dabei auf gewöhnlichen Sonntagsgottesdiensten. Die zwei Arten von Daten - objektive und subjektive - werden anschliessend verglichen. Als dritter Punkt werden die Einstellungen der Pfarrpersonen zu Dialekt und Standarddeutsch präsentiert.

\subsection{Mittels Code-Switching den Gottesdienst gestalten}

Die Verfügbarkeit von zwei Varietäten - Dialekt und Standarddeutsch - führt dazu, dass in vielen Gottesdiensten (meist mehrfach) zwischen diesen hin- und hergewechselt wird. Solche Code-Switchings ${ }^{8}$ kommen im Korpus der Gottesdienstaufnahmen zahlreich und vor allem in beide Richtungen vor.

Aus soziopragmatischer Perspektive kommt Code-Switching eine funktionale Bedeutung zu (cf. z. B. Gardner-Chloros 2009, Gumperz 1982, Lüdi 2004). Insbesondere das ,diskursbezogene Code-Switching“, das Auer (2009: 93) beschreibt, ist eine für die vorliegende Studie relevante Kontextualisierungsstrategie. Die in den untersuchten Gottesdiensten analysierten Code-Switchings lassen den Schluss zu, dass solche Wechsel ein multifunktionales Instrument der Gottesdienstgestaltung darstellen. Wechsel sind in beide Richtungen möglich. Beim Wechsel vom Dialekt zu Standarddeutsch werden Funktionen der Schriftlichkeit aktiviert:

- Zitieren bzw. Pseudozitieren aus der Schriftlichkeit (z. B. Bibelverse, Liedtitel, Redewendungen)

- Vorlesen/Ablesen von schriftlichen Vorlagen (z. B. Bibeltexte, liturgische Texte, literarische Texte)

- Propria erkennbar machen (Veranstaltungstitel)

Bei diesen Wechseln zu Standarddeutsch wird der „sozialsymbolische Wert“ dieser Varietät „als authentischer [...] Grösse ( $\rightarrow$ Verweis auf das sprachliche Original)“ (Christen et al.

\footnotetext{
${ }^{8}$ Bereits Gumperz' (1982: 59) Definition lässt die Interpretation zu, nicht nur Wechsel zwischen Sprachen als Code-Switching zu interpretieren, sondern auch solche zwischen Standardsprache und Dialekten. Gemäss Christen et al. (2010: 57) kann es ,mittlerweile als unbestritten gelten, dass CS [= Code-Switching; S.O.] nicht nur zwischen etablierten Standardvarietäten, sondern auch zwischen Standardsprachen und Dialekten oder zwischen einzelnen Dialekten vorkommen kann".
} 
2010: 95) aktiviert. In der Domäne der Schriftlichkeit hält Standarddeutsch in der Deutschschweiz relativ unbestritten seine Stellung. ${ }^{9}$ Daher erstaunt dieser Gebrauch von Standarddeutsch im Gottesdienst, insbesondere für das Zitieren schriftlicher Originale, nicht. Die folgenden beiden Beispiele - eines aus einer katholischen Messe, eines aus einem reformierten Gottesdienst - illustrieren solche Code-Switchings. Beim Bsp. 1 handelt es sich um den Übergang von der Einleitung des Vaterunsers zum Vaterunser, das in seiner standarddeutschen Fassung gesprochen wird:

Bsp. 1):

zu gott wo üüs nööch isch | törfe mer voll vertruue mitenand bätte $\mid<$ pause> (1.0) | vater unser im himmel | geheiligt werde dein name [Pfarrperson, k005005, Einleitung zum Vaterunser $>$ Vaterunser $]^{10}$

Einen weiteren Wechsel vom Dialekt ins Standarddeutsche während der Predigt ist in Bsp. 2 zu sehen. Dieses Code-Switching demonstriert das Potential der Diglossie auf exemplarische Weise:

Bsp. 2):

me söll nöd säge chumm daas mache mer etzt immer esoo | oder miir gönd immer döthii oder miir mached aifach ales mitenand daa isch soo | söll me nöd säge oder leered miir | aber i de bible staat $\mid$ alle dinge ooni usnaam sind möglich dem der glaubt $\mid$ soo es vols muul $\mid<$ pause > (1.6) | ali sache | eben aa die wo miir nöd draa tänked dass sii vilicht möglich wääred [Pfarrperson r004005, Predigt] ${ }^{11}$

Das Bibelzitat wird durch den Wechsel zu Standarddeutsch markiert. Mitten im Zitat wechselt die Pfarrperson jedoch wieder zum Dialekt, um eine Ergänzung des Zitats durch ihre eigene Gedanken zu markieren. Das Muster, dem hier gefolgt wird, kann kurz zusammengefasst werden: Alles, was Standarddeutsch ist, sind fremde Worte, alles, was im Dialekt gesprochen wird, eigene.

Die Funktionen von Code-Switchings vom Standarddeutschen in den Dialekt sind hingegen vielfältiger: Die Wechsel aktivieren Funktionen der Mündlichkeit, d. h. der Domäne des Dialekts in der Deutschschweiz, können Dramaturgieeffekte (wie Regieanweisung, Perspektivenwechsel, Schlusssignal, inszenierte Spontaneität) erzielen oder sie sind der realen Spontaneität (Autokorrektur, Kommentar, Entschuldigung) geschuldet. Darüber hinaus können auch Schweizer Eigenheiten so markiert und Propria erkennbar gemacht werden.

Bsp. 3 zeigt einen solchen Wechsel von Standarddeutsch in den Dialekt während des Abendmahls eines reformierten Gottesdienstes:

\footnotetext{
${ }^{9}$ Ausnahmen sind Kontexte, in denen der Dialekt als schriftliche Varietät verwendet wird, um konzeptionelle Mündlichkeit zu markieren (z. B. SMS und seine Nachfolger, Chats, E-Mails, cf. z. B. Stähli et al. 2011).

${ }^{10} \mathrm{Zu}$ Gott, der uns nah ist, dürfen wir voller Vertrauen miteinander beten.

${ }^{11}$ Man soll nicht sagen: Komm, wir machen das jetzt immer so. Oder: Wir gehen immer dorthin. Oder: Wir machen einfach alles miteinander, das ist so. Soll man nicht sagen. Oder lernen wir. Aber in der Bibel steht: [...] ohne Ausnahme [...]. So einen vollen Mund. Alle Sachen, eben auch die, an die wir nicht denken, dass sie möglich wären.
} 


\section{Bsp. 3):}

christus hat zu seinen aposteln gesagt | frieden hinterlasse ich euch | meinen frieden gebe ich euch vilicht das au miir denand $\mid<$ pause $>(0.6) \mid$ es spüürbaars zaiche vo dem fride | jetzt wiitergänd | <pause, geräusche, flüsternde stimmen> (32.5) | kommt es ist alles bereit [Pfarrperson r001001, Abendmahl Friedensgruss] ${ }^{12}$

Dieser Wechsel ist ein Beispiel von inszenierter Spontaneität (villicht „vielleicht“): Es gibt keine Pause zwischen der Standarddeutsch gesprochenen Passage und dem Dialekt, es kann sich also nicht um eine spontane Idee der Pfarrperson handeln. Darüber hinaus markiert die Pfarrperson so einen Perspektivenwechsel und begibt sich von der verkündenden Rolle auf die Ebene der Gemeinde (miir ,wir“) und gibt zusätzlich eine Regieanweisung im Dialekt.

Dasselbe macht der katholische Pfarrer in Bsp. 4; er markiert die Regieanweisung zur Ausführung des Friedensgrusses durch einen Wechsel in den Dialekt:

Bsp. 4):

christus ist unser friede und unsere versöhnung deshalb bitten wir $\mid<$ pause $>$ (1.6) $\mid$ herr jesus christus $\mid<$ pause $>$ (1.2) | schau nicht auf unsere sünden $\mid$ sondern auf den glauben deiner kirche | und schenke nach deinem willen | einheit und frieden | der friede des herrn sei allezeit mit euch $\mid$ und mit deinem geiste ${ }^{13}$ | gämer enand es zaiche vom fride und vo de versöönig | <pause, geräusche> (23.4) [Pfarrperson k004004, Einleitung Friedensgruss $>$ Friedensgruss $]^{14}$

Die Diglossie ermöglicht den Pfarrpersonen also das bewusste Einsetzen der beiden Varietäten und bildet somit eine kommunikative Ressource sowohl im reformierten wie auch im katholischen Gottesdienst, die von den Pfarrerinnen und Pfarrern entsprechend genutzt wird. Es lässt sich also feststellen, dass gerade die Verfügbarkeit von zwei Varietäten den spezifischen Sprachgebrauch von Deutschschweizer Pfarrpersonen massgebend prägt.

\subsection{Verschiedene Varietätenmuster im reformierten Gottesdienst möglich}

Die 40 Tonaufnahmen von reformierten Sonntagsgottesdiensten lassen sich sechs verschiedenen Varietätenmustern zuordnen. Die Zuteilung $\mathrm{zu}$ diesen Mustern geschah aufgrund der für die einzelnen Gottesdienstteile gewählten Varietät. Wie in Tab. 1 ersichtlich wird, kommen in 33 der Gottesdienste Code-Switchings vor. An welchen Stellen diese auftauchen und welche Rolle diese bei der Gottesdienstgestaltung einnehmen, wird in Kapitel 4.3 ausgeführt. Zunächst wird hier ein Blick auf die vorkommenden Varietätenmuster geworfen:

\footnotetext{
12 Vielleicht, dass auch wir einander ein spürbares Zeichen von diesem Frieden jetzt weitergeben.

${ }^{13}$ Kursive Passage von der Gemeinde und der Pfarrperson gemeinsam gesprochen.

${ }^{14}$ Geben wir einander ein Zeichen vom Frieden und von der Versöhnung.
} 


\begin{tabular}{|l|l|}
\hline Varietätenmuster & $\begin{array}{l}\text { Anzahl } \\
\text { Gottesdienste }\end{array}$ \\
\hline 1) Standardgottesdienst & 7 \\
\hline $\begin{array}{l}\text { 2) Standardgottesdienst mit wenigen dialektalen Passagen (z. B. für Taufe } \\
\text { oder Mitteilungen) }\end{array}$ & 12 \\
\hline $\begin{array}{l}\text { 3) Dialekt-Standarddeutschgottesdienste I: Dialekt für frei Formuliertes, } \\
\text { Standarddeutsch für übernommenen Text }\end{array}$ & 8 \\
\hline $\begin{array}{l}\text { 4) Dialekt-Standarddeutschgottesdienste II: wie Muster 3, aber mit kleine- } \\
\text { ren Abweichungen (z. B. standarddeutsche Predigt, dialektales Eingangs- } \\
\text { wort) }\end{array}$ & 9 \\
\hline 5) Dialektgottesdienst mit marginalem Standarddeutschanteil & 2 \\
\hline 6) Mischformen ohne klar erkennbare Varietätenverteilung & 2 \\
\hline
\end{tabular}

Tabelle 1: Verteilung der 40 reformierten Gottesdienste auf sechs Varietätenmuster

Lediglich sieben Gottesdienste werden in Standarddeutsch ohne Code-Switchings zum Dialekt abgehalten; in weiteren 12 dominiert Standarddeutsch relativ klar. Das bedeutet, dass rund die Hälfte der untersuchten Gottesdienste (47.5\%) ausschliesslich oder grossmehrheitlich in Standarddeutsch gefeiert wird.

Dieses Bild relativiert sich aber, wenn die eigenen Aufnahmen und die Vergleichsaufnahmen getrennt betrachtet werden. Abb. 3 zeigt, dass die Muster 1 und 2 bei den Vergleichsaufnahmen weit stärker vertreten sind als bei den eigenen Aufnahmen. Dies liegt daran, dass in einer der beiden Kirchgemeinden, von der die Aufnahmen stammen, im Predigtgottesdienst gemäss schriftlicher Regelung „Hochdeutsch“ verwendet werden soll und die meisten untersuchten Aufnahmen aus dieser Gemeinde Predigt- bzw. Festgottesdienste dokumentieren. Die Pfarrpersonen halten sich offensichtlich an diese Vorgabe:

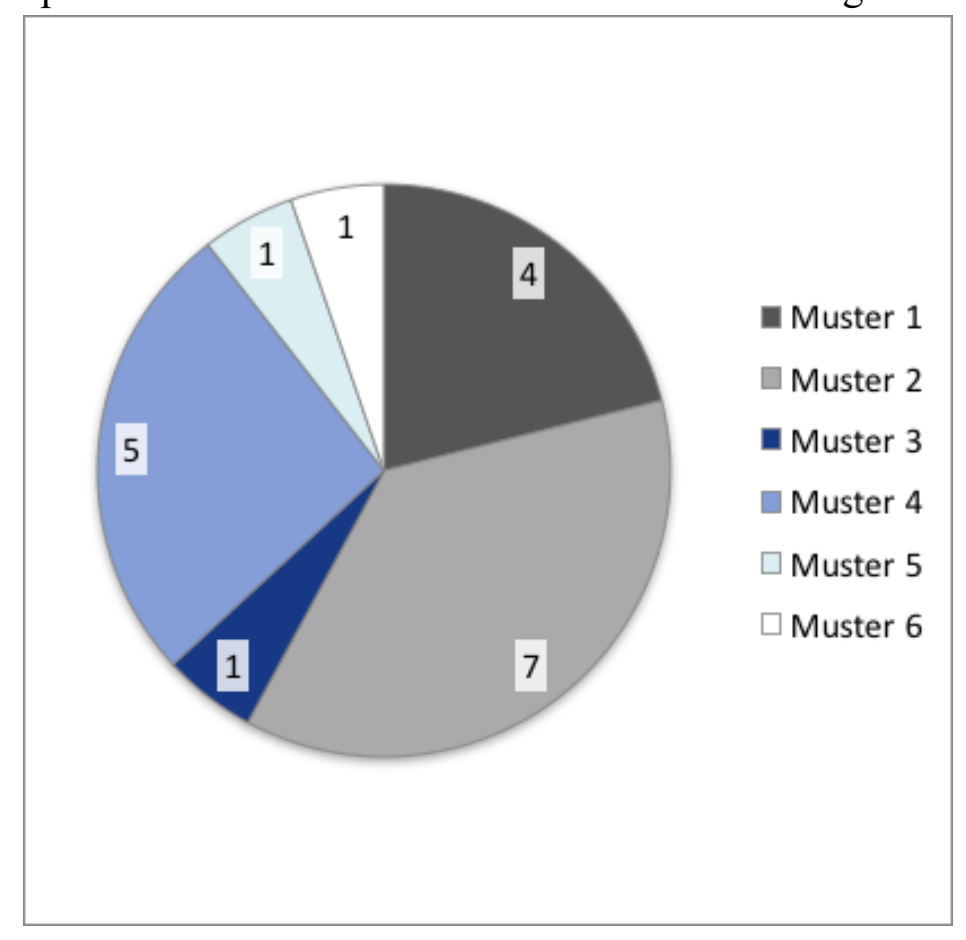

Abbildung 3: Varietätenmuster in den Vergleichsaufnahmen (Zahlen geben die Anzahl der Gottesdienste an) 
Bei den eigenen Aufnahmen zeigt sich eine Präferenz für die Muster 3 und 4, jedoch ist die Quote der Gottesdienste, die ausschliesslich oder grossmehrheitlich in Standarddeutsch gehalten werden, mit 38,1\% immer noch sehr hoch:

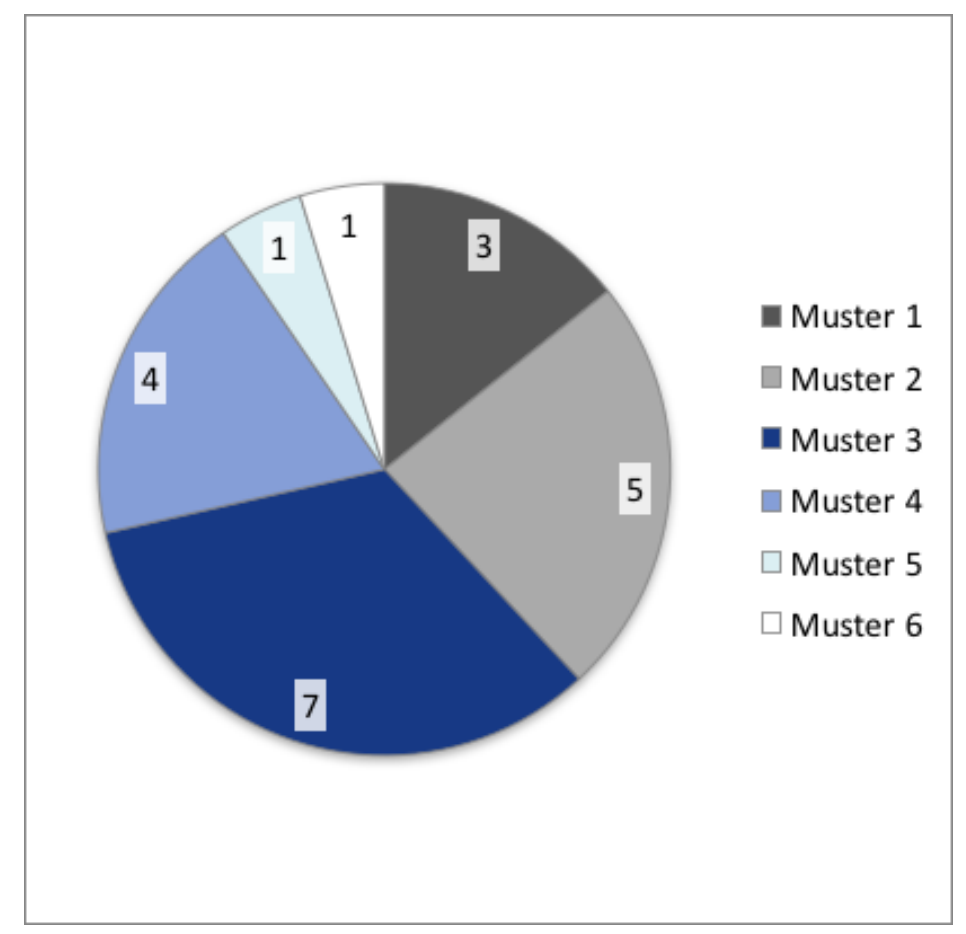

Abbildung 4: Varietätenmuster in den eigenen Gottesdienstaufnahmen

Die Familiengottesdienste verteilen sich auf die (eher) dialektal geprägten Muster 4 und 5; hier ist Standarddeutsch offensichtlich nicht so gefragt. Die Festgottesdienste sind ebenfalls in der Mehrheit dialektal geprägt. Dies erstaunt, da die Meinung vorherrscht, dass gerade der Grad der höheren Feierlichkeit Standarddeutsch nach sich ziehen könnte (cf. z. B. Bietenhard 1990: 188, Rüegger et al. 1996: 110, Kamm 2007: 14f.). ${ }^{15}$ Im vorliegenden Korpus lässt sich diese Gleichung aber nicht machen. Am stärksten gegen Dialekt resistent zeigen sich die Predigtgottesdienste.

Der Anteil von standarddeutsch geprägten Gottesdiensten ist in beiden Korpusteilen recht hoch angesichts der Tatsache, dass in der Literatur allenthalben vom Ausbreiten des Dialekts in Gottesdiensten die Rede ist (cf. z. B. Kap. 2). Nicht zuletzt deswegen ist ein Blick auf die gewählte Varietät für die einzelnen Teile des Gottesdienstes spannend. Hier lassen sich grosse Unterschiede feststellen:

\footnotetext{
15 David Plüss, Professor für Praktische Theologie an der Universität Bern, sprach im Interview, das für die Studie durchgeführt wurde, ebenfalls vom feierlicheren Charakter der Standardsprache: „Also grundsätzlich oder in der Tendenz ist Standardsprache feierlicher, wenn sie verdichtet ist, wenn sie geprägt ist, wenn sie auch alt ist, wenn man es merkt, dass sie alt ist, als eine Sprache, die nach Alltag, nach Small Talk tönt.“ Er gab aber gleichzeitig zu bedenken, dass die Gleichung Feierlichkeit = Standarddeutsch so nicht automatisch gemacht werden könne, weil auch eine Kinderweihnacht im Dialekt durchaus feierlich sein könne.
} 


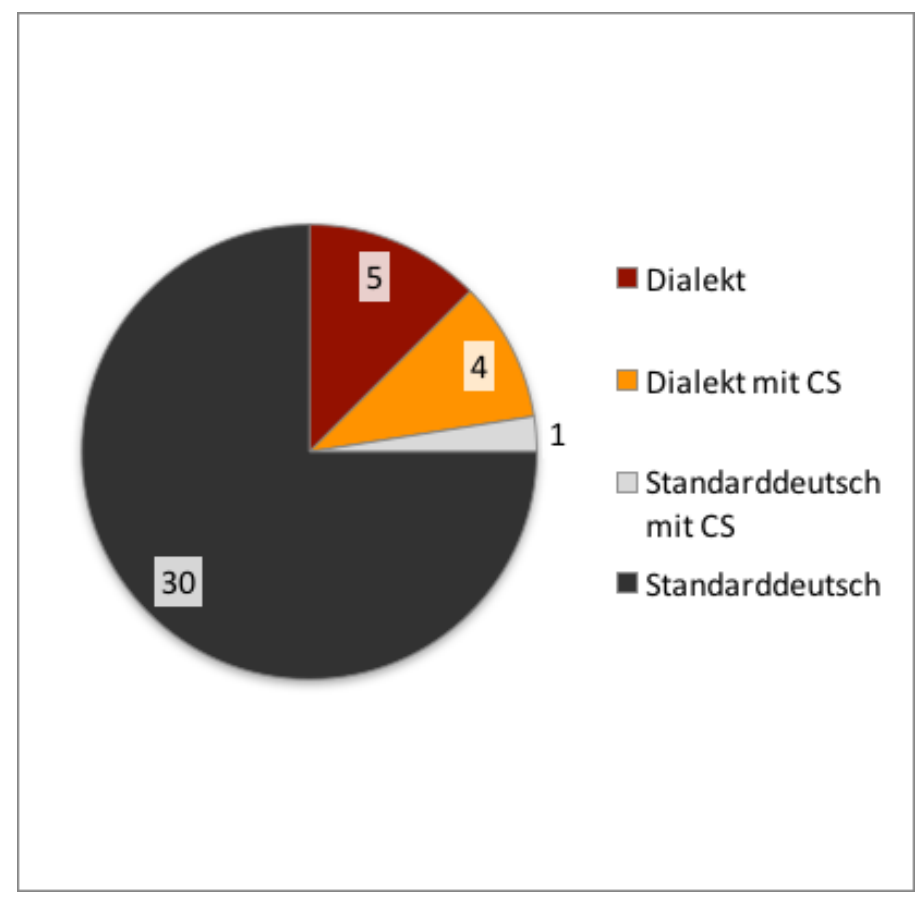

Abbildung 5: Eingangswort der reformierten Gottesdienste nach gewählter Varietät (n=40)

Das Eingangswort wird zu $75 \%$ in Standarddeutsch realisiert; in einem knappen Viertel der Gottesdienste wird dafür der Dialekt, allenfalls mit Code-Switching, gewählt (cf. Abb. 5). Dieses Resultat ist wenig erstaunlich, weil das Eingangswort in der Regel aus einem Bibelvers besteht, der „originalgetreu“ in Standarddeutsch gelesen/gesprochen wird. Ganz anders sieht es bei den Mitteilungen aus, einem sehr frei formulierten Gottesdienstteil: Hier werden der Gemeinde beispielsweise Veranstaltungen der Kirchgemeinde angekündigt, der Bestimmungszweck der Kollekte mitgeteilt oder sonstige Mitteilungen gemacht. Diese erfolgen zu zwei Dritteln im Dialekt (allenfalls mit Code-Switching fürs Zitieren von Veranstaltungstiteln o. Ä.). Nur in einem Viertel der Gottesdienste werden die Mitteilungen ausschliesslich in Standarddeutsch gesprochen.

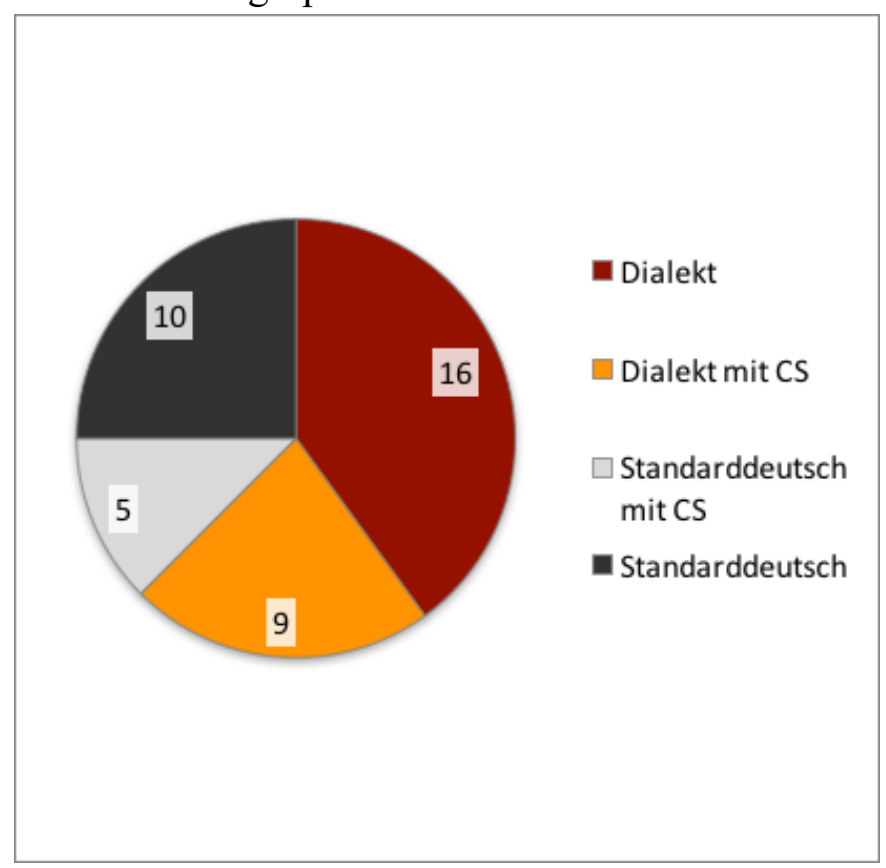

Abbildung 6: Mitteilungen der reformierten Gottesdienste nach gewählter Varietät (n=40) 
Es lässt sich feststellen, dass der Sprachgebrauch im reformierten Gottesdienst sich häufig am Muster orientiert: für fremde Worte Standarddeutsch - für selbst formulierte Texte Dialekt. Dies zeigt sich bei einem Blick auf die Varietätenwahl für die einzelnen Gottesdienstteile. Code-Switchings kommen sowohl an den Grenzen zwischen zwei Gottesdienstteilen (Bsp. 1, Bsp. 4) vor, von denen einer fremde Worte (z. B. Eingangswort) und einer eigene Worte (z. B. Begrüssung) enthält, als auch innerhalb einzelner Gottesdienstteile (Bsp. 2, Bsp. 3). Insgesamt ist der Anteil von Standarddeutsch höher, als dies aufgrund der Ausgangshypothese, dass Standarddeutsch durch den Dialekt zurückgedrängt würde, die auf den Schilderungen in der Literatur fusste, zu erwarten war.

\subsection{Dialekt als mögliche Varietät für nicht rituelle Teile katholischer Gottesdienste}

Nachdem sich bei den reformierten Gottesdiensten sechs mögliche Varietätenmuster abzeichnen, lassen sich solche Aussagen für das kleine Sample an katholischen Gottesdiensten nicht treffen. Dennoch sind gewisse Muster betreffend den Varietätengebrauch zu erkennen: Von den sechs analysierten katholischen Gottesdiensten kommt keiner ohne Code-Switching in den Dialekt aus. Es dominiert jedoch klar das Standarddeutsche in allen sechs Messen. Fast ausschliesslich Standarddeutsch gesprochen wird das Hochgebet: Der Dialekt findet sich dort höchstens in Form von Liedeinleitungen oder der Einleitung zum Vaterunser. Ausserhalb des Hochgebets gibt es für den Dialekt offensichtlich mehr Spielraum: So können die Einführung und die Verabschiedung, die Einleitungen zu Liedern und anderen Liturgieteilen (Ausnahme: Lesungen, Gebete) im Dialekt gesprochen werden. Darüber hinaus wird in drei der Gottesdienste die Predigt im Dialekt gehalten.

Es wird klar, dass die rituellen (teils gemeinsam gesprochenen) Texte standarddeutsch bleiben. Diese Teile des Gottesdienstes bieten auch allochthonen Gottesdienstbesucherinnen und -besuchern die Möglichkeit, sich in der katholischen Messe „zu Hause zu fühlen“: Wären diese im Dialekt gesprochen, wäre die Gefahr grösser, solche Personen auszuschliessen, da das Rituelle den eigentlichen Kern der katholischen Messe darstellt. Die weniger stark ritualisierten Teile können hingegen im Dialekt gesprochen werden, ohne dass der Charakter einer katholischen Messe für allochthone Personen verloren geht. Es lässt sich also feststellen, dass je höher die Ritualität eines Teils der katholischen Messe bzw. einer Formel innerhalb der Messe ist, diese/r umso stärker mit Standarddeutsch verbunden ist. Wo hingegen die Ritualität in der Tendenz gering(er) ist, findet der Dialekt den Weg auch in die katholische Messe. ${ }^{16}$

Die Funktionen von Code-Switchings im katholischen Sample entsprechen den für die reformierten Gottesdienste festgestellten: Es konnten Wechsel zum Markieren von Zitaten, des Gottesdienstschlusses sowie von Regieanweisungen festgestellt werden. Auch Perspektivenwechsel, die durch Varietätenwechsel angezeigt werden, finden sich im Korpus.

\footnotetext{
16 Lötscher (1983: 130) glaubte, dass als Folge des „Liturgiewandels“ des Zweiten Vatikanischen Konzils „vielerorts sozusagen ein direkter Wechsel vom Latein zur Mundart [erfolgte]“. So massiv stellt sich der Wechsel in den sechs untersuchten katholischen Gottesdiensten jedoch nicht dar.
} 


\subsection{Intendierter Varietätengebrauch im reformierten Gottesdienst}

Sowohl die Pfarrpersonen im Interview als auch die reformierten Pfarrpersonen in der Fragebogenerhebung wurden nach ihrem intendierten Varietätengebrauch für den Sonntagsgottesdienst und weitere Gottesdienste gefragt. Im Folgenden werden zuerst die Daten zu den „gewöhnlichen Sonntagsgottesdiensten“ präsentiert, anschliessend jene zu Abdankungsgottesdiensten. ${ }^{17}$

\subsubsection{Sonntagsgottesdienste: Zweiteilung nach Art des gesprochenen Textes}

Es zeigt sich, dass die Daten von Interviews und Fragebögen bezüglich des intendierten Varietätengebrauchs im gewöhnlichen Sonntagsgottesdienst weitgehend übereinstimmen, wie hier am Beispiel der Gottesdienstteile Eingangswort (Abb. 7) und Begrüssung (Abb. 8) illustriert wird:

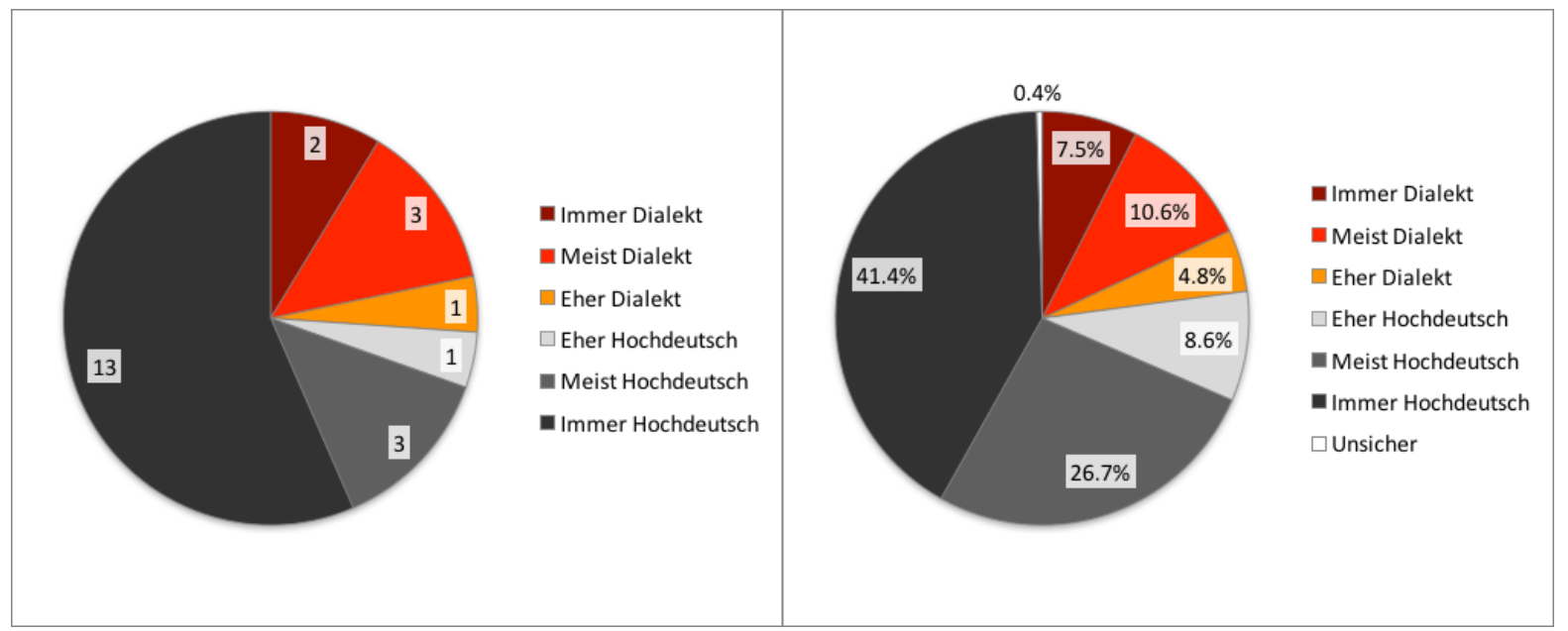

Abbildung 7: Intendierter Varietätengebrauch für das Eingangswort: Interviews (links, n=23) und Fragebögen (rechts, $n=454$ ) im Vergleich

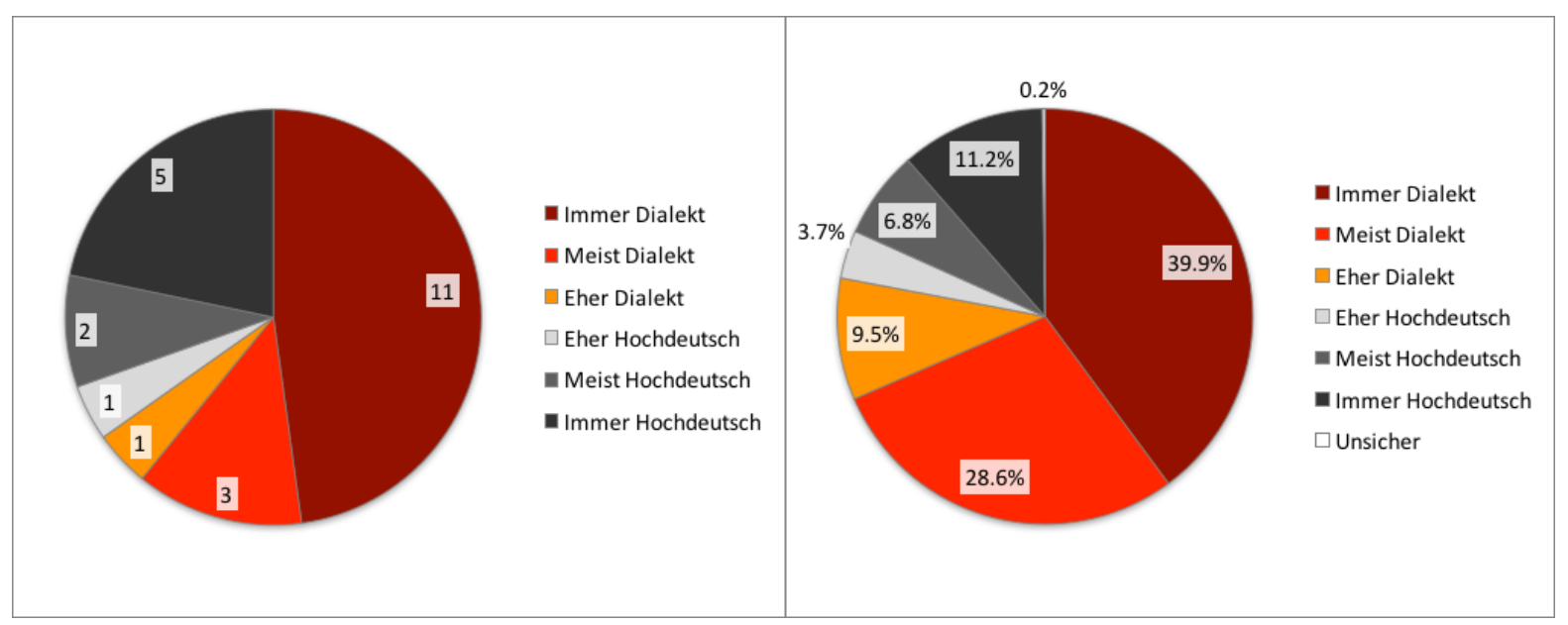

Abbildung 8: Intendierter Varietätengebrauch für die Begrüssung: Interviews (links, n=23) und Fragebögen (rechts, $n=454$ ) im Vergleich

Die Pfarrpersonen verhalten sich also gemäss eigener Aussage ähnlich, wenn man die Antworten „Immer Hochdeutsch“, „Meist Hochdeutsch“ und „Eher Hochdeutsch“ bzw. die entsprechenden Antworten für den Dialekt zusammenzählt. Es ergibt sich beim Eingangswort

17 „Gottesdienst für Verstorbene anlässlich des Begräbnisses“ cf. Variantenwörterbuch (Ammon et al. 2016: 4). 
eine zwei-Drittel- bis drei-Viertel-Präferenz für das Hochdeutsche, bei der Begrüssung zahlenmässig eine ähnliche Präferenz in die umgekehrte Richtung. Da die Begrüssung auf das Eingangswort folgt, scheint hier ein Übergang zu sein, wo Code-Switchings in vielen Fällen stattfinden.

Standarddeutsch scheint also auch im Bewusstsein der Pfarrpersonen für die eigene Varietätenwahl je nach Gottesdienstteil nach wie vor eine wichtige Rolle zu spielen, sodass sich hier das Bild der objektiven Sprachdaten durch die subjektive Einschätzung der Sprecherinnen und Sprecher zu verfestigen scheint.

\subsubsection{Abdankungsgottesdienste stärker dialektal geprägt}

Die Pfarrpersonen äusserten sich in den Interviews und Fragebögen nicht nur zum (intendierten) Sprachgebrauch im Predigtgottesdienst, sondern auch zu jenem in anderen Gottesdienstformen sowie weiteren kirchlichen Kontexten. Als Beispiel für eine andere Art von Gottesdienst soll hier der Abdankungsgottesdienst anhand der Daten der Gewährspersonen aus der Fragebogenerhebung dienen. Die Pfarrpersonen kreuzten bei der Fragebogenerhebung pro Teil eines solchen Gottesdienstes die Varietät an (in den bereits oben erwähnten Abstufungen „,immer“, „meist“, „,eher"). Zählt man die Werte für Hochdeutsch bzw. Dialekt zusammen und vergleicht diese mit jenen des Sonntagsgottesdienstes, zeigt sich für die Abdankungen eine Tendenz hin zu mehr Dialekt:

\begin{tabular}{|l|l|l|}
\hline Gottesdienstteil & Abdankung & Sonntagsgottesdienst \\
\hline Eingangswort & $35.8 \%$ & $22.9 \%$ \\
\hline Begrüssung & $89.9 \%$ & $78.0 \%$ \\
\hline Liedansagen & $90.5 \%$ & $78.0 \%$ \\
\hline Eingang/Ausgang der Lesung & $84.4 \%$ & $71.6 \%$ \\
\hline Lesung & $8.1 \%$ & $4.6 \%$ \\
\hline Predigt & $87.2 \%$ & $69.4 \%$ \\
\hline Gebete (ohne Unser Vater und Fürbitte) & $79.5 \%$ & $66.3 \%$ \\
\hline Unser Vater & $1.6 \%$ & $0.4 \%$ \\
\hline Mitteilungen/Kollekte & $90.4 \%$ & $78.6 \%$ \\
\hline Sendungswort & $37.9 \%$ & $26.9 \%$ \\
\hline Segen & $22.5 \%$ & $15.6 \%$ \\
\hline
\end{tabular}

Tabelle 2: Präferenz für Dialekt für die einzelnen Gottesdienstteile (zusammengezählte Werte) in Abdankungs- und Sonntagsgottesdiensten im Vergleich $(n=454)$

Die Werte für den Dialekt sind durchgängig höher für den Abdankungsgottesdienst, sowohl bei den Gottesdienstteilen, die sehr tiefe Werte aufweisen (Unser Vater, Lesung), als auch bei jenen, die bereits für den Sonntagsgottesdienst hohe Werte zeigen (Mitteilungen, Liedansagen, Begrüssung). Es könnte sein, dass sich die Pfarrpersonen bei der sprachlichen Gestaltung von Abdankungsgottesdiensten von den expliziten Wünschen der Trauerfamilien leiten lassen: $82.2 \%$ der Pfarrpersonen fragen gemäss eigenen Angaben die Trauerfamilie vor der Beerdigung nach der bevorzugten Sprachform.

Auch die Angaben aus den Interviews zu Taufen, sowohl von katholischen wie reformierten Pfarrpersonen, weisen auf einen stärkeren Dialektgebrauch für Kasualgottesdienste hin: Von 
den 24 reformierten Pfarrpersonen geben 17 an, die Taufen im Dialekt zu halten, von den 6 Katholiken geben 4 an, sehr viel Dialekt für Taufgottesdienste zu verwenden, die anderen beiden verwenden gleich viel Dialekt wie in Sonntagsgottesdiensten, welche gemäss dem von ihnen angekreuzten Schema bereits viel Dialekt enthalten.

Der Dialekt scheint in Kasualien also wichtiger zu sein als in regulären Sonntagsgottesdiensten; diese Tendenz ist aber keine neue: Gemäss Schwarzenbach (1969: 225) hatte die Dialektpredigt bereits in den Sechzigerjahren in dieser Art von Gottesdiensten ,einen bestimmten festen Platz erworben." Laut den Angaben der Pfarrpersonen im Fragebogen geht diese Wirkung aber über die Predigt hinaus.

\subsection{Intendierter und tatsächlicher Varietätengebrauch im Vergleich}

Interessant ist nun ein Vergleich von objektiven und subjektiven Daten der reformierten Pfarrpersonen: Stimmt die subjektive Einschätzung mit dem tatsächlichen Sprachgebrauch überein? Aufgrund der Diglossiesituation in der Deutschschweiz müssen sich die Sprecherinnen und Sprecher entscheiden, welche Varietät sie in solch einem Kontext, wo aufgrund der Möglichkeit des situationsinduzierten Standardgebrauchs sowohl Dialekt als auch Standarddeutsch zur Verfügung stehen, sprechen. Dieses Faktum bringt Pfarrperson k001001 im Interview wie folgt auf den Punkt: „Ich kann nicht mich nicht entscheiden als Seelsorger. Ich muss mir überlegen, wo rede ich Hochdeutsch und wo Schweizerdeutsch."18

Die folgenden Gegenüberstellungen von Gottesdienstteilen zeigen, dass Pfarrpersonen offensichtlich sehr sprachbewusste Sprecherinnen und Sprecher sind. Das intendierte und das tatsächliche Sprachverhalten stimmen weitgehend überein. ${ }^{19}$ Die objektiven Daten sind in vier Kategorien eingeteilt (Gottesdienstteile, die ausschliesslich in Dialekt oder Standarddeutsch gehalten wurden, und solche, bei denen Dialekt bzw. Standarddeutsch dominiert, aber mindestens ein Code-Switching in die andere Varietät vorkommt), die subjektiven Daten in sechs Kategorien (,immer“, „meist“, „eher“ für jede Varietät). In den Diagrammen sind die dialektal geprägten Kategorien in Rottönen eingefärbt, die standarddeutsch geprägten in Grautönen.

Die Einschätzung und das tatsächliche Verhalten decken sich bei fast allen Gottesdienstteilen (cf. die Abb. 9 und 10 als illustrierende Beispiele).

18 Die Tiefeninterviews wurden im Dialekt geführt, da sowohl die Interviewten wie die Interviewerin autochthone Dialektsprecher sind. Ein Wechsel zum Standarddeutschen hätte gegen die pragmatische Regel verstossen, dass Deutschschweizerinnen und Deutschschweizer untereinander in informellen Situationen den Dialekt verwenden (cf. Haas 1998: 78). Transliteriert wurden die Interviews aber aus praktischen Gründen in Standarddeutsch.

${ }^{19}$ Natürlich können hier auch Abweichungen stattfinden: So hielt Pfarrperson r008009 ihren Gottesdienst komplett in Standarddeutsch (im Dialekt waren lediglich die Lieder, die eine Band vortrug), kreuzte aber beim Interview für die Begrüssung, die Liedansagen, die Abkündigungen, das Fürbittegebet und die Mitteilungen ,meist Dialekt“ und für die Gebete „eher Dialekt“ an. Sie begründete ihr Verhalten im aufgezeichneten Gottesdienst bereits zu Beginn des Interviews (ohne dass die Rede schon auf ihr Sprachverhalten im Gottesdienst gekommen war): „Also eben, an dem Sonntag, an dem Sie gekommen sind, sind ja Asylbewerber im Gottesdienst gewesen und darum habe ich dann den ganzen Gottesdienst auf Hochdeutsch zu machen probiert, obwohl ich eigentlich die Liturgie normalerweise schweizerdeutsch mache.“

ISSN 1615-3014 


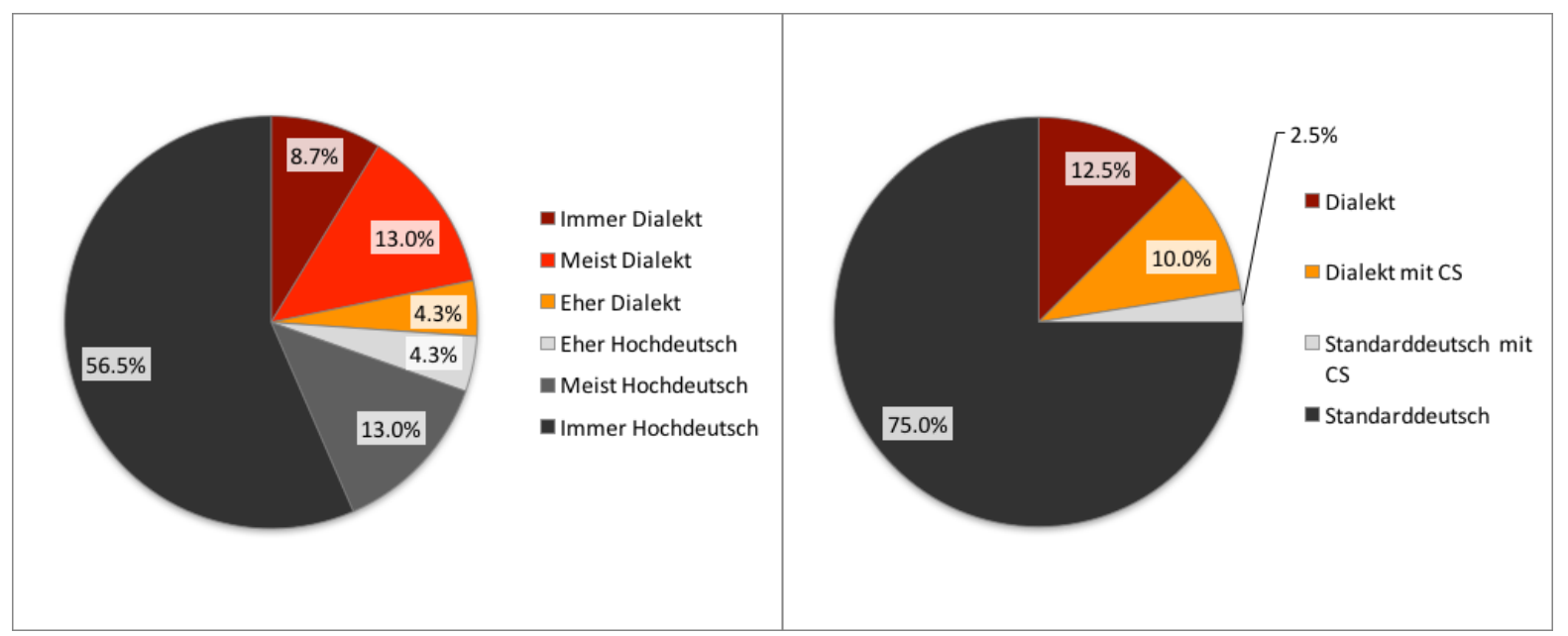

Abbildung 9: Subjektive (links, n=23) und objektive Daten (rechts, $n=40$ ) im Vergleich für das Eingangswort

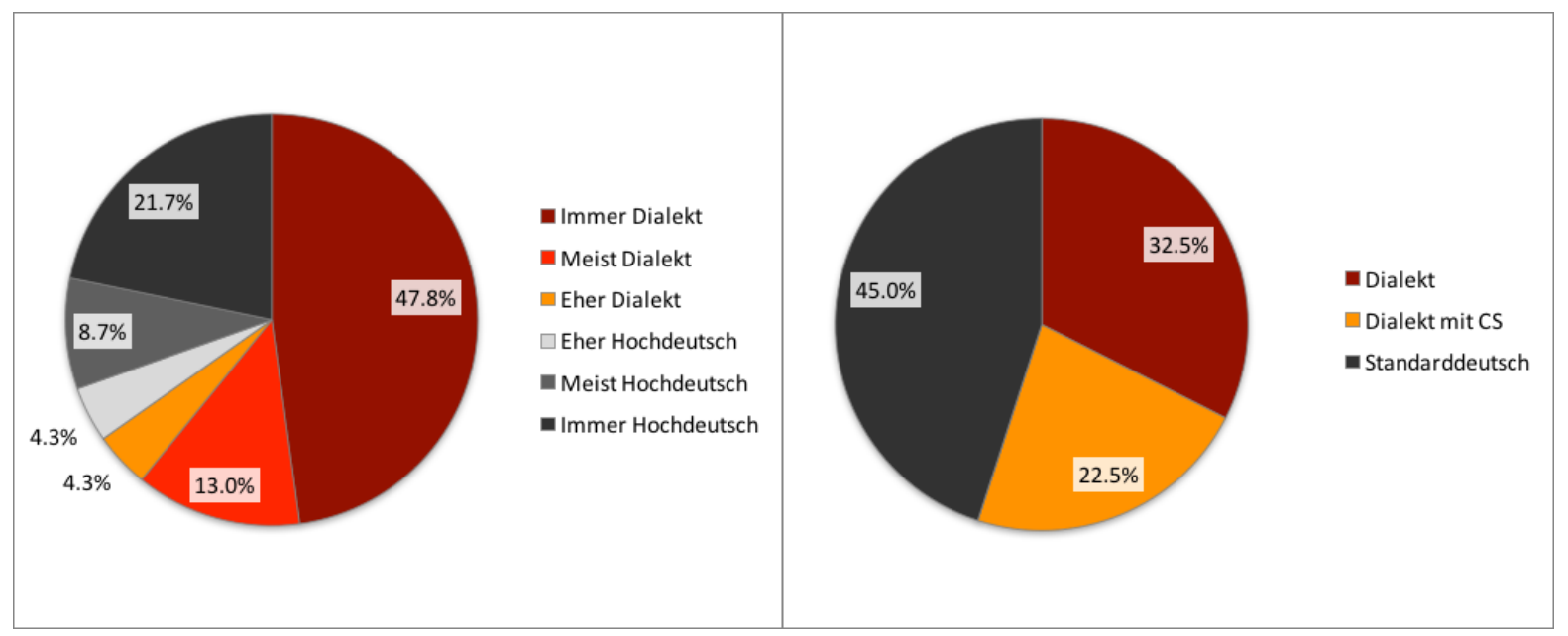

Abbildung 10: Subjektive (links, $n=23$ ) und objektive Daten (rechts, $n=40$ ) im Vergleich für die Begrüssung

Ein leicht abweichendes Bild zeigt sich lediglich für einen Gottesdienstteil: die Gebete (cf. Abb. 11). Hier ist im Korpus der Gottesdienstaufnahmen der Standarddeutsch-Anteil höher. Dies könnte aber mit den Vergleichsaufnahmen, die stärker standarddeutsch geprägt sind, zusammenhängen (cf. Kap. 4.2). 


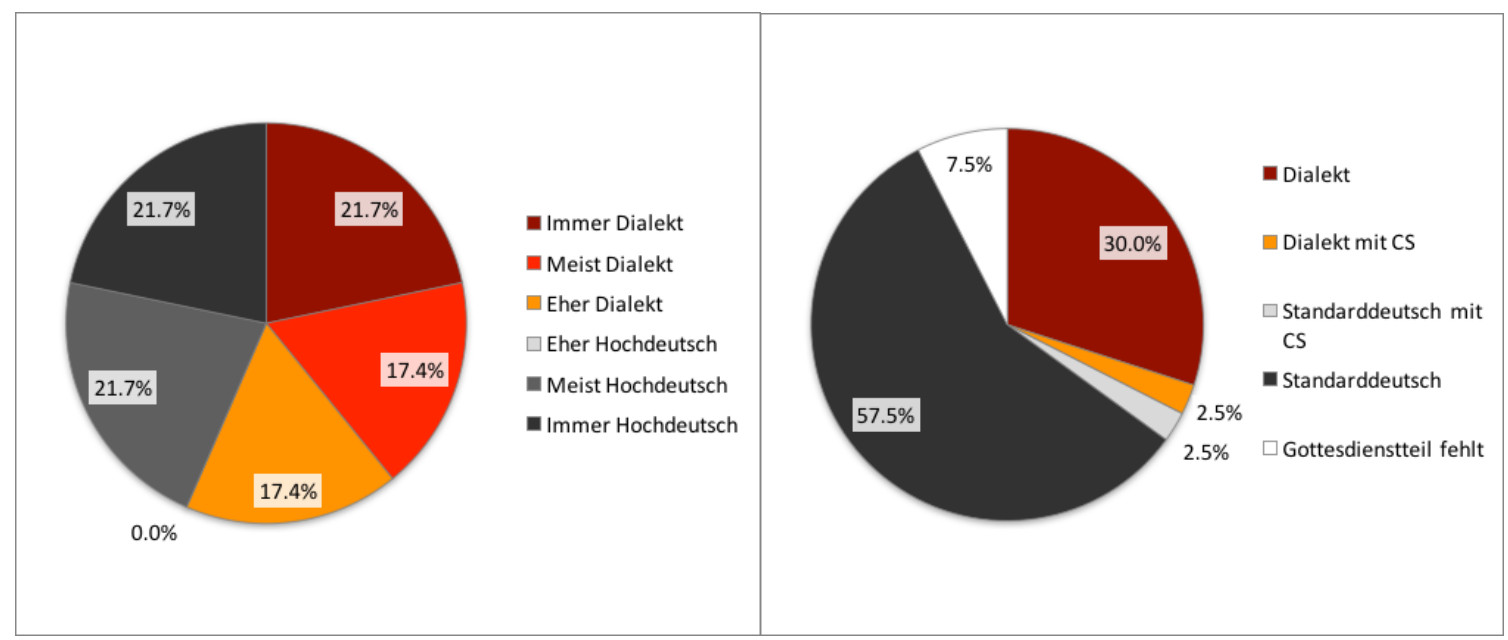

Abbildung 11: Subjektive (links, n=23) und objektive Daten (rechts, $n=40$ ) im Vergleich für das Gebet (für die Aufnahmen wurde nur das jeweils erste Gebet in die Auswertung einbezogen)

Den Gesamteindruck von sehr sprachbewussten (Berufs-)Sprecherinnen und -sprechern, die genau wissen, wie sie sich sprachlich verhalten, kann dieser Ausreisser jedoch nicht trüben.

\subsection{Differenzierte Spracheinstellungen zu Dialekt und Standarddeutsch erkennbar}

Als letzter Punkt sollen hier die Spracheinstellungen zu Dialekt und Hochdeutsch 20 thematisiert werden. Bisherige Studien erweckten bisweilen den Eindruck, dass Deutschweizerinnen und Deutschschweizer tendenziell negative Einstellungen $\mathrm{zu}$ Hochdeutsch haben - oder zumindest ein sehr gespaltenes bzw. widersprüchliches Verhältnis zu dieser Varietät (cf. z. B. Gutzwiller 1991, Scharloth 2005, Hove 2002). Christen et al. (2010: 15) gehen davon aus, dass die Widersprüchlichkeiten in der Bewertung darin begründet sind, dass ,die erfragte Grösse ,Hochdeutsch“ kein einheitliches Konzept ist, sondern sich aus verschiedenen mentalen Modellen zusammensetzt" und je nach Frage ein anderes Modell ,,aktiviert““ wird. Sie erwähnen vier mögliche Modelle: A) Hochdeutsch als plurizentrische Sprache, B) Hochdeutsch als kodifizierte Grösse/(Standard-)Norm, C) Hochdeutsch als Lese- und Schreibsprache, D) Hochdeutsch als lebendige Alltagssprache (cf. Christen et al. 2010: 15, Formulierung nach Sieber 2013: 122). ${ }^{21}$ In Anlehnung an diese Hypothese von Christen et al. wurde in der vorliegenden Studie versucht, im Interview verschiedene Hochdeutsch- und auch Dialektmodelle zu aktivieren, so z. B. „Hochdeutsch als Sprache in der Schweiz“, „Hochdeutsch als Sprache im Kindergarten“, „Hochdeutsch als Sprache in der Schule“ oder auch „Hochdeutsch als Gottesdienst- und Predigtsprache“. Damit sollte geprüft werden, inwiefern die Beurteilung der beiden Sprachformen konsistent ist und ob Hochdeutsch tatsächlich mehrheitlich negativ eingeschätzt wird.

\footnotetext{
${ }^{20}$ Der Terminus „Hochdeutsch“ wird hier verwendet, wenn von den subjektiven Meinungen der Sprecherinnen und Sprechern die Rede ist.

${ }^{21}$ Für Sieber (2013: 123) sind „,weitere mentale Modelle von Hochdeutsch [denkbar], die je nach Verwendungssituationen, sprachlicher Sozialisation und kulturellen Orientierungen zusätzliche Aspekte von Hochdeutsch in den Fokus rücken können (z. B. Mehrsprachigkeit, Berufsspezifika, künstlerische Orientierungen etc.)“. Eine solche Ergänzung um weitere Modelle wurde für die hier vorgestellte Studie vorgenommen.
}

ISSN 1615-3014 


\subsubsection{Erwartbare Stereotype mit erstaunlichem Resultat für Hochdeutsch}

Die Resultate zeigen zunächst, dass die erwarteten (und bereits hinlänglich bekannten) Stereotype zu den beiden Varietäten genannt werden, wenn man die Pfarrpersonen nach einem Vergleich der beiden fragt: ${ }^{22}$ So wird der Dialekt im Vergleich zu Hochdeutsch in der Fragebogenerhebung wie folgt beschrieben (offene Fragestellung, kategorisierte Antworten, die drei häufigsten Antwortkategorien):

- $\quad 29.3 \% \quad$ „näher, nah, Nähe, weniger distanziert“

- $\quad 20.3 \% \quad$ „gefühlsbetont(er), gefühlvoller, emotional(er)“

- $19.3 \% \quad$ „Herzenssprache, Sprache des Herzens“

Hochdeutsch wird im Vergleich zum Dialekt charakterisiert als:

- $\quad 27.3 \% \quad$ „präzise(r), genau(er), exakt(er)“

- $22.9 \% \quad$ „distanziert(er), fern(er), schafft Distanz“

- $20.3 \% \quad$ „Schriftsprache, Sprache des Schreibens“

Mit $11.7 \%$ Nennungen folgt „Fremdsprache“ erst auf Platz 5 (umgekehrt ist „Mutter-/ Vatersprache mit $16.3 \%$ beim Dialekt-Hochdeutsch-Vergleich auf Platz 4). Diese Prozentzahl erscheint doch sehr tief angesichts der Annahme, dass Hochdeutsch für Deutschschweizerinnen und Deutschschweizer eine Fremdsprache sein soll. ${ }^{23}$ Offensichtlich herrscht zumindest bei der hier befragten Gruppe von Deutschschweizerinnen und Deutschschweizern diese Meinung nicht mehrheitlich vor.

Die Beurteilungen zeigen im Gesamten in Richtung einer - wie erwartet - klar positiven Bewertung des Dialekts und in Richtung einer etwas distanzierteren, aber tendenziell positiver als erwarteten Einschätzung von Hochdeutsch. Dies könnte damit zusammenhängen, dass Pfarrpersonen in ihrer Ausbildung und im beruflichem Alltag überdurchschnittlich häufig mit Hochdeutsch zu tun haben und diese Varietät deswegen möglicherweise bereits in der auf Stereotype abzielenden Gegenüberstellung der Sprachformen positiver bewerten als andere Schweizerinnen und Schweizer.

\footnotetext{
22 Sieber (2013: 120) weist darauf hin, dass, ,,was die Einschätzung der beiden Varietäten Mundarten und Hochdeutsch betrifft, in Beschreibung und Diskussion immer wieder die gleichen Stereotypen [sic!] referiert und damit auch verfestigt“" werden. So wird eben der Dialekt als ,persönlich, vertraut, locker, frei, einfach, ausdrucksstark, sympathisch und lustig“ eingeschätzt, während Standarddeutsch mit „,unpersönlich, unvertraut, steif, kompliziert, wenig emotional, gepflegt und gehoben" assoziiert werde (Sieber/Sitta 1986: 122, Hervorhebung im Original).

23 Berthele (2004: 127; Hervorhebung im Original Fettdruck) schreibt beispielweise, dass Standarddeutsch „nach meiner Beobachtung spontan nicht nur einfach als andere Sprache als der Dialekt bezeichnet [wird], sondern mehrheitlich sogar als Fremdsprache“. Von den Befragten in Scharloths Studie glauben 79 \%, dass Hochdeutsch für Deutschschweizer die erste Fremdsprache sei (cf. Scharloth 2005: 240). Er geht davon aus, dass sich „[d]ieser Befund [...] mit den Ergebnissen früherer Studien [deckt], die die Einstellungen der Deutschschweizer zum Standarddeutschen in den Blick nahmen: Sie konstatieren ausnahmslos negative Haltungen dem Hochdeutschen gegenüber" (Scharloth 2005: 241).
} 


\subsubsection{Differenzierte Einstellungen dank Konzept von Hochdeutschmodellen}

Die Antworten auf verschiedene Fragen, die auf unterschiedliche Hochdeutschmodelle abzielten, zeigen, dass neben den stereotypen Beurteilungen der beiden Sprachformen, mittels derer sich Pfarrpersonen auch innerhalb der Deutschschweizer Sprachgemeinschaft positionieren, ${ }^{24}$ sehr differenzierte Einstellungen zu beiden Sprachformen vorhanden sind. Bei den meisten der untersuchten 30 Pfarrpersonen werden offensichtlich verschiedene mentale Modelle von Hochdeutsch (und auch Dialekt) in den Köpfen aktiviert, also verschiedene Komponenten der Beurteilung der Varietät angesprochen. ${ }^{25}$ Dies soll an vier Beispielen kurz aufgezeigt werden:

1) Pfarrperson r016018 hält Hochdeutsch für eine Fremdsprache, ,eine gelernte Sprache, in der ich auch probiere, Fortschritte zu machen". Gleichzeitig ist Hochdeutsch aber auch „völkerverbindend“ und für sie selbstverständliche Gottesdienstsprache in einer Gemeinde in einem Tourismusgebiet. Die Varietät habe zudem den Vorteil der Verständlichkeit als Gottesdienstsprache. Jedoch findet sie es „ein bisschen früh“, wenn im Kindergarten schon Hochdeutsch gesprochen wird.

2) Pfarrperson r001001 hält Hochdeutsch für die „viel nuancenreicher[e], viel reichhaltiger[e]“ Varietät. Hochdeutsch habe „etwas von neutral an sich“. Ausserdem helfe Hochdeutsch bei der Integration Anderssprachiger. Gleichzeitig findet die reformierte Pfarrperson, es sei übertrieben, im Kindergarten sowie im Turn- und Zeichenunterricht Hochdeutsch zu sprechen. Auch für den Religionsunterricht erscheint ihr Dialekt als die bessere Varietät. Als Gottesdienstsprache habe Hochdeutsch den Vorteil, „dass man sich differenzierter ausdrücken kann“. Der Fall von r001001 zeigt ausserdem die Wichtigkeit vom Miteinbezug sprachlicher Biographien, weil diese auch mögliche Veränderungen in den Einstellungen sichtbar machen und erklären (cf. auch Lenz 2003: 266): Die Pfarrperson hat längere Zeit in Deutschland studiert und gibt an, dass Hochdeutsch die Familiensprache ist: Sie spricht mit dem Ehepartner und den (erwachsenen) Kindern so.

3) Dasselbe gilt für den katholischen Priester k001001, der ein Jahr seiner Ausbildung in Österreich aboslviert hat und seine Einstellungen zu Hochdeutsch aufgrund der Erfahrungen in dieser Zeit offensichtlich verändert hat, wie das folgende Interviewzitat zeigt:

Hochdeutsch, also ich kann mich gut erinnern, als ich nach [Ort in Österreich] gekommen bin, also das erste Mal eine längere Zeit im Ausland, im deutschsprachigen Raum, das ist für mich unglaublich anstrengend gewesen, Hochdeutsch zu reden. Also ich habe das sehr anstrengend gefunden, auch im Alltag alles am Frühstückstisch schon auf Hochdeutsch zu sagen. Und nach diesem Jahr ist das für mich also völlig normal. [...] Und ich verstehe Leute bei uns, die das einfach anstrengend finden, Hochdeutsch zu reden. Für meinen Vater ist das anstrengend. Das ist eine Art eine Fremdsprache. (Pause) Aber es ist eben eine, eine grosse Kultursprache, es eröffnet mir einen riesigen Kulturraum, Literatur, Theater, Poesie, Philosophie, Begriffe auch, die gefüllt sind mit einem Hintergrund (Pause). Also wo ich gar kein Verständnis habe, wenn irgendwelche Leute sich lustig machen über einen Schweizer Akzent im Hochdeutschen, also das mag ich gar nicht mehr hören, weil (Pause) ich finde, das darf man hören, woher jemand kommt, und man hört es de facto auch. [...]

(Pfarrperson k001001)

\footnotetext{
${ }^{24}$ Cf. z. B. Reisigl (2008) und Garrett (2010) zur identitätsstiftenden Funktion von Stereotypen.

${ }^{25}$ Für eine Zusammenstellung der Einstellungen aller untersuchten Pfarrpersonen cf. Oberholzer (in Vorb.).
} 
Hochdeutsch ist für k001001 selbst also keine Fremdsprache mehr, er versteht aber die Einschätzung als Fremdsprache durch andere Deutschschweizer Personen. Gleichzeitig verfügt er über ein ausgeprägtes Plurizentrizitätsbewusstsein und fordert die Plurizentrizität dementsprechend selbstbewusst ein. Er verbindet die Varietät auch mit verschiedenen Bereichen der Kultur. Im Kindergarten befürwortet er eine Koexistenz der beiden Varietäten, in der Schule soll klar Hochdeutsch die wichtigere Rolle spielen.

4) Pfarrperson r010012 bewertet hingegen Hochdeutsch in allen angesprochenen Kontexten tendenziell eher negativ; es wirke im Vergleich zum Dialekt „so ein bisschen hochgestochener teilweise, und eben ein bisschen distanzierter“. Den Gebrauch von Hochdeutsch im Kindergarten lehnt sie ab, ebenso steht sie der uneingeschränkten Verwendung von Hochdeutsch in der Schule kritisch gegenüber. Gefragt nach den spezifischen Leistungen von Standarddeutsch als Gottesdienst- und Predigtsprache findet die Pfarrrperson, es gebe aus ihrer Sicht keine, ,ausser eben vielleicht, wenn man nicht genau weiss, hat es Leute drin, die es nicht verstehen“. Bei r010012 handelt es sich um die einzige der untersuchten Personen, die Hochdeutsch gegenüber relativ grundsätzlich negativ eingestellt ist und bei der diese Einschätzung während des Interviews konstant bleibt.

Die vier erwähnten Beispiele von Deutschschweizer Pfarrpersonen zeigen, dass das Ansprechen verschiedener Hochdeutschmodelle einen vielversprechenden Zugang zu Spracheinstellungen in der Deutschschweiz darstellt: Es wird klar, dass je nach Frage verschiedene Modelle aktiviert werden und diese $\mathrm{zu}$ unterschiedlichen Beurteilungen der Varietät führen, die weit über die bekannten stereotypen Bewertungen hinausgehen. Dabei können sowohl sehr konsistent negative Einschätzungen vorkommen (wie bei r010012) als auch heterogene Bewertungen (r016018, r001001, k001001) auftreten. Wichtig erscheint in jedem Fall, (Sprach-)Biographien miteinzubeziehen, da diese mögliche Erklärungen für veränderte Einstellungen liefern und sowohl sehr positive wie auch sehr negative Einschätzungen mitprägen können.

\section{Diglossie als Mehrwert und Standarddeutsch als Potential}

Deutschschweizerinnen und Deutschschweizern wird nachgesagt, dass sie nicht gerne Hochdeutsch sprechen und Hochdeutsch als (erste) Fremdsprache empfinden. Diese Meinung gehört zu den gängigen Sprachformenstereotypen in der Schweiz und taucht auch in Kommentarspalten von Zeitungen (Onlineportale) immer wieder auf:

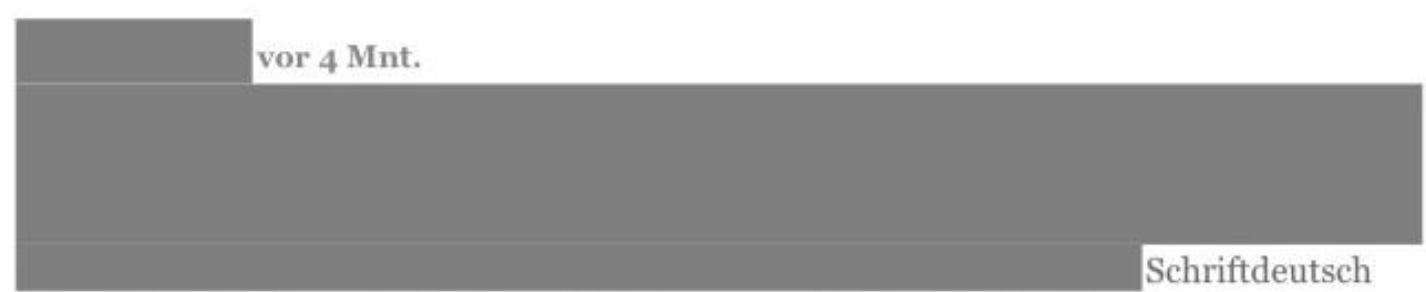

stellt für die allermeisten Kinder eine Fremdsprache dar, da dauert es eine Weile, bis das sitzt.

- Empfehlen (11) Melden Teilen Antworten

Abbildung 12: Kommentar auf www.tagesanzeiger.ch zum Artikel „Deutsch und uneindeutig“ (TagesAnzeiger 2016, zitiert am 2.9.2016)

ISSN 1615-3014 
In diesem Beitrag wurde aufgezeigt, dass es viel $\mathrm{zu}$ kurz greift, die gängigen Spracheinstellungen von Deutschschweizerinnen und Deutschschweizern auf diese zwei Aussagen zu reduzieren. ${ }^{26}$ Die Berufsgruppe, die im Zentrum der vorgestellten Studie steht, besteht aus sehr sprachaffinen und sprachbewussten Sprecherinnen und Sprechern. Die untersuchten Pfarrpersonen der reformierten und katholischen Kirche verfügen durch die Diglossie über ein probates Mittel, ihre Gottesdienste sprachlich zu gestalten: Die beiden Varietäten Schweizerdeutsch und Standarddeutsch sind funktional einsetzbar; CodeSwitchings haben das Potential, verschiedene Funktionen auszudrücken. Am häufigsten wird dabei Standarddeutsch dort eingesetzt, wo ein Zitat aus der Schriftlichkeit markiert werden soll. Standarddeutsch bietet sich dafür selbstverständlich an, da es in der Deutschschweiz im Bereich der Schriftlichkeit nach wie vor relativ unbestritten die natürlich verwendete Sprachform ist. Es kann zumindest für den Kontext Kirche nicht behauptet werden, dass der Dialekt alleinige und stark vorherrschende Varietät ist: Die Resultate der Studie zeigen vielmehr, dass es vom Gottesdienstteil und auch der Gottesdienstart abhängig ist, welche Varietät gesprochen wird. Die beiden Sprachformen werden offensichtlich funktional eingesetzt, was die Annahme stützt, dass die Sprachsituation in der Deutschschweiz zurecht als Diglossie beschrieben wird. Die Diglossie stellt für Deutschschweizer Sprecherinnen und Sprecher einen kommunikativen Mehrwert dar. Standarddeutsch ist darüber hinaus als gesprochene Varietät in der Schweiz eine wichtige kommunikative Ressource, die zumindest von der hier untersuchten Berufsgruppe - bewusst genutzt wird und deren Wert nicht unterschätzt werden darf.

Der Blick auf den intendierten Sprachgebrauch bestätigt, dass Pfarrpersonen sehr bewusste Sprachbenutzerinnen und -benutzer sind: Die subjektive Einschätzung stimmt mit der objektiv festgestellten Varietätenverwendung weitgehend überein. Die Sprecherinnen und Sprecher wissen also sehr genau, wann sie welche Sprachform in ihren Gottesdiensten einsetzen. Darüber hinaus konnten die Einschätzungen der 24 interviewten reformierten Pfarrpersonen durch die Resultate aus einer breit angelegten Fragebogenerhebung mit 454 Gewährspersonen validiert werden. Das Muster für die Varietätenverwendung scheint Folgendes zu sein: Alles, was eigenes Gedankengut ist, wird tendenziell im Dialekt gesprochen. Für alles, was Worte von anderen sind, wird Standarddeutsch verwendet.

Schliesslich konnte gezeigt werden, dass die in der Deutschschweiz gängigen Sprachformenstereotype auch Pfarrpersonen bekannt sind, sie aber in der Tendenz Standarddeutsch positiver einschätzen, als dies erwartet wurde. Insbesondere hat das Fremdsprachen-Stereotyp kein überragendes Gewicht. Nur $11.7 \%$ der Befragten nannten dieses in der Onlinebefragung. In den Tiefeninterviews wurden zudem verschiedene Hochdeutschmodelle anzusprechen versucht: Die Resultate zeigen, dass dieser Weg ein aufschlussreiches Bild von Spracheinstellungen zutage fördert. Je nach angesprochenem Modell beurteilen die Gewährspersonen die Varietäten potentiell unterschiedlich und konstruieren so sehr vielschichtige, mehrdimensionale Einstellungen zu Dialekt und Hochdeutsch. Es zeigt sich klar, dass die gängige Meinung, dass Deutschschweizerinnen und Deutschschweizer Hochdeutsch als erste Fremdsprache empfinden, nicht gern sprechen und tendenziell negativ beurteilen, zumindest für die hier untersuchte Sprechergruppe keine

${ }^{26}$ Cf. auch Christen et al. (2010) für den Standardgebrauch von Deutschschweizer Polizistinnen und Polizisten.

ISSN 1615-3014 
Gültigkeit hat. Vielmehr lassen sich je nach angesprochenem Hochdeutschmodell klar positive Einstellungen zur Standardvarietät feststellen.

Angesichts dieser Resultate wird klar, dass nicht pauschal von den Spracheinstellungen der Deutschschweizerinnen und Deutschschweizern gesprochen werden kann, sondern offensichtlich sehr verschiedene Einstellungsmuster zu Dialekt und Hochdeutsch vorhanden sind, die jedoch in der Tendenz eine viel stärker positive Beurteilung von Hochdeutsch erkennen lassen, als dies bisher für Deutschschweizer Sprecherinnen und Sprecher bekannt war. Für die künftige Spracheinstellungsforschung in der Deutschschweiz erscheint der Ansatz, mehrere mentale Hochdeutschmodelle anzunehmen, lohnenswert. Auch der Miteinbezug sprachlicher Biographien hilft dabei, bisherige eingeschränkte Sichtweisen auf Spracheinstellungen von Deutschschweizerinnen und Deutschschweizern zu verlassen und den Fokus stärker auf die Mehrdimensionalität solcher Einschätzungen zu legen. Damit könnten bisher vorhandene, tendenziell negative Stereotype gegenüber Standarddeutsch mittelfristig aufgebrochen und durch positivere, die den realen Einschätzungen möglicherweise eher entsprechen, ersetzt werden.

\section{Literaturverzeichnis}

Auer, Peter (2009): „Competence in performance: Code-switching und andere Formen bilingualen Sprechens“. In: Gogolin, Ingrid/Neumann, Ursula (eds.): Streitfall Zweisprachigkeit - The Bilingualism Controversy. Wiesbaden, VS Verlag für Sozialwissenschaften: 91-110.

Bachmann, Thomas/Good, Bruno (2003): Hochdeutsch als Unterrichtssprache. Befunde und Perspektiven. Zürich: Bildungsdirektion/Volksschulamt.

Bachmann, Thomas/Sigg, Marianne (2004): Hochdeutsch-Kindergarten: Die Chancen des frühen Beginns. Bericht zur explorativen Studie „Hochdeutsch im Übergang zwischen Kindergarten und Primarschule“. Zürich: Pädagogische Hochschule Zürich.

Berthele, Raphael (2004): „Vor lauter Linguisten die Sprache nicht mehr sehen - Diglossie und Ideologie in der deutschsprachigen Schweiz“. In: Christen, Helen (ed.): Dialekt, Regiolekt und Standardsprache im sozialen und zeitlichen Raum. Beiträge zum 1. Kongress der Internationalen Gesellschaft für Dialektologie des Deutschen, Marburg/Lahn, 5.-8. März 2003. Wien, Edition Praesens: 111-136.

Berthele, Raphael (2010): „Dialekt als Problem oder Potenzial? Überlegungen zur Hochdeutschoffensive in der deutschen Schweiz aus Sicht der Mehrsprachigkeitsforschung“. In: Bitter Bättig, Franziska/Tanner, Albert (eds.): Sprachen lernen - durch Sprache lernen. Zürich, Seismo: 37-52.

Bietenhard, Hans (1990): „Die Sprache der Kirche. Gedanken eines Bibelübersetzers“. In: Vouga, Jean-Pierre/Hodel, Max Ernst (eds.): La Suisse face à ses langues = Die Schweiz im Spiegel ihrer Sprachen = La Svizzera e le sue lingue. Aarau, Sauerländer: 185-190.

Christen, Helen et al. (2010): Hochdeutsch in aller Munde. Eine empirische Untersuchung zur gesprochenen Standardsprache in der Deutschschweiz. Stuttgart: Steiner. (=Zeitschrift für Dialektologie und Linguistik. Beihefte 140).

Gardner-Chloros, Penelope (2009): Code-switching. Cambridge: Cambridge University Press. Garrett, Peter (2010): Attitudes to language. Cambridge: Cambridge University Press. (=Key topics in sociolinguistics 9). 
Gumperz, John J. (1982): Discourse strategies. Cambridge: Cambridge University Press. (=Studies in interactional sociolinguistics 1).

Gutzwiller, Jürg (1991): „Identität versus Kommunikation. Junge Deutschschweizer zwischen Dialekt und Standardsprache“. In: Schläpfer, Robert et al. (eds.): Das Spannungsfeld zwischen Mundart und Standardsprache in der deutschen Schweiz. Spracheinstellungen junger Deutsch- und Welschschweizer. Eine Auswertung der Pädagogischen Rekrutenprüfungen 1985. Aarau, Sauerländer: 45-214. (=Pädagogische Rekrutenprüfungen 12).

Gyger, Mathilde (2005): Projekt Standardsprache im Kindergarten (PSS). Schlussbericht. Basel: Rektorat Kindergärten Basel, Arbeitsgruppe Integration.

Haas, Walter (1998): „Diglossie im historischen Wandel oder: Schweizerdeutsch auf dem Weg zur eigenen Sprache?“. In: Russ, Charles V.J. (ed.): Sprache Kultur Nation. Language Culture Nation. Papers from a conference held on 19 October 1996 at the Guildhall, York. Hull, University of Hull: 77-101. (=New German Studies Texts \& Monographs XII).

Haas, Walter (2000): „Die deutschsprachige Schweiz“. In: Schläpfer, Robert/Bickel, Hans (eds.): Die viersprachige Schweiz. Aarau, Sauerländer: 57-138.

Haas, Walter (2004): „Die Sprachsituation der deutschen Schweiz und das Konzept der Diglossie“. In: Christen, Helen (ed.): Dialekt, Regiolekt und Standardsprache im sozialen und zeitlichen Raum. Beiträge zum 1. Kongress der Internationalen Gesellschaft für Dialektologie des Deutschen, Marburg/Lahn, 5.-8. März 2003. Wien, Edition Praesens: 81110.

Hove, Ingrid (2002): Die Aussprache der Standardsprache in der deutschen Schweiz. Tübingen: Niemeyer. (=Phonai 47).

Koch, Kurt (1990): „Dialekt oder Hochsprache im Gottesdienst der christlichen Kirchen?“. In: Vouga, Jean-Pierre/Hodel, Max Ernst (eds.): La Suisse face à ses langues = Die Schweiz im Spiegel ihrer Sprachen = La Svizzera e le sue lingue. Aarau, Sauerländer: 182184.

Lenz, Alexandra N. (2003): Struktur und Dynamik des Substandards. Eine Studie zum Westmitteldeutschen (Wittlich/Eifel). Stuttgart: Steiner. (=Zeitschrift für Dialektologie und Linguistik. Beihefte 125).

Lötscher, Andreas (1983): Schweizerdeutsch. Geschichte, Dialekte, Gebrauch. Frauenfeld: Huber.

Lüdi, Georges (2004): „Code-Switching“. In: Ammon, Ulrich et al. (eds.): Sociolinguistics. An International Handbook of the Science of Language and Society. 2., vollständig neu bearbeitete und erweiterte Auflage. Berlin/New York, de Gruyter: 341-350. (=HSK 3.1).

Meili, Ernst (1985): „Wort und Sprache in der reformierten Kirche“. In: Arens, Peter et al. (eds.): Des Schweizers Deutsch. Bern, Hallwag: 104-136.

Oberholzer, Susanne (2006): „Schweizer Hochdeutsch als Unterrichtssprache. Am Beispiel des Kantons Thurgau“. In: Dürscheid, Christa/Businger, Martin (eds.): Schweizer Standarddeutsch. Beiträge zur Varietätenlinguistik. Tübingen, Narr: 233-263.

Oberholzer, Susanne (in Vorb.): Zwischen Standarddeutsch und Dialekt. Untersuchung zu Sprachgebrauch und Spracheinstellungen von Pfarrpersonen in der Deutschschweiz. Stuttgart: Steiner. (=Zeitschrift für Dialektologie und Linguistik. Beihefte). 
Petkova, Marina (2012): „Die Deutschschweizer Diglossie. Eine Kategorie mit fuzzy boundaries". Dinge und Maschinen in der Kommunikation. Zeitschrift für Literaturwissenschaft und Linguistik 42/168: 126-154.

Rash, Felicity (1998): The German Language in Switzerland. Multilingualism, Diglossia and Variation. Bern etc.: Peter Lang. (=German Linguistic and Cultural Studies 3).

Reisigl, Martin (2008): Stereotyp. Ein ambiges Konzept zwischen verfestigter Denkökonomie, sprachlichem Schematismus und gefährlicher Handlungsdetermination [I]. Archiv für Begriffsgeschichte 50: 231-253.

Rickenmann, Agnell (2005): „Mundart und Kirche“. In: Barblan, Paolo/Koller, Arnold (eds.): Dialekt in der (Deutsch)Schweiz - Zwischen lokaler Identität und nationaler Kohäsion. Menziken, Forum Helveticum: 118-124.

Ris, Roland (1990): „Diglossie und Bilingualismus in der deutschen Schweiz: Verirrung oder Chance?“. In: Vouga, Jean-Pierre/Hodel, Max Ernst (eds.): La Suisse face à ses langues = Die Schweiz im Spiegel ihrer Sprachen = La Svizzera e le sue lingue. Aarau, Sauerländer: 40-49.

Rüegger, Beat et al. (1996): Mundart und Standardsprache im reformierten Gottesdienst. Eine Zürcher Untersuchung. Aarau: Sauerländer. (=Reihe Sprachlandschaft 18).

Scharloth, Joachim (2005): „Asymmetrische Plurizentrizität und Sprachbewusstsein. Einstellungen der Deutschschweizer zum Standarddeutschen“. Zeitschrift für Germanistische Linguistik 33/2: 236-267.

Schläpfer, Robert (1994): „Mundart und Standardsprache“. In: Bickel, Hans/Schläpfer, Robert (eds.): Mehrsprachigkeit - eine Herausforderung. Aarau, Sauerländer: 281-296. (=Reihe Sprachlandschaft 13).

Schwarzenbach, Rudolf (1969): Die Stellung der Mundart in der deutschsprachigen Schweiz. Studien zum Sprachbrauch der Gegenwart. Frauenfeld: Huber.

Siebenhaar, Beat/Wyler, Alfred (1997): Dialekt und Hochsprache in der deutschsprachigen Schweiz [Vorabdruck]. 5., vollständig überarbeitete Auflage. Zürich: Pro Helvetia.

Sieber, Peter (2010): „Deutsch in der Schweiz: Standard, regionale und dialektale Variation“. In: Krumm, Hans-Jürgen et al. (eds.): Deutsch als Fremd- und Zweitsprache. Berlin/New York, de Gruyter: 372-385. (=HSK 35.1).

Sieber, Peter (2013): „Probleme und Chancen der Diglossie - Einstellungen zu Mundarten und Hochdeutsch in der Deutschschweiz“. In: Eriksson, Brigit et al. (eds.): Sprechen und Zuhören - gefragte Kompetenzen? Überzeugungen zu Mündlichkeit in Schule und Beruf. Bern, hep: 106-136. (=Mündlichkeit 2).

Sieber, Peter/Sitta, Horst (1986): Mundart und Standardsprache als Problem der Schule. Aarau: Sauerländer. (=Reihe Sprachlandschaft 3).

Steck, Wolfgang (1988): „Kasualien“. In: Balz, Horst Robert et al. (eds.): Theologische Realenzyklopädie. Jesus Christus V-Katechismuspredigt. Band 17. Berlin/New York, de Gruyter: 673-686.

Urwyler, André/Gysel, Irene (2011): „Dialekt oder Standardsprache?“. In: Kunz, Ralph et al. (eds.): Reformierte Liturgik - kontrovers. Zürich, Theologischer Verlag Zürich: 299-318. (=Praktische Theologie im reformierten Kontext 1$)$. 